\title{
An invitation to toric degenerations
}

\author{
Mark Gross and Bernd Siebert
}

\section{Contents}

Introduction $\quad 43$

1. Purely toric constructions 44

2. Introducing singular affine structures $\quad 50$

3. Examples without scattering 54

4. Scattering 63

$\begin{array}{ll}\text { 5. Three-dimensional examples } & 72\end{array}$

$\begin{array}{ll}\text { References } & 77\end{array}$

\section{Introduction}

In $[\mathbf{G r S i 2}]$ we gave a canonical construction of degenerating families of varieties with effective anticanonical bundle. The central fibre $X$ of such a degeneration is a union of toric varieties, glued pairwise torically along toric prime divisors. In particular, the notion of toric strata makes sense on the central fiber. A somewhat complementary feature of our degeneration is their toroidal nature near the 0-dimensional toric strata of $X$; near these points the degeneration is locally analytically or in the étale topology given by a monomial on an affine toric variety. Thus in this local model the central fiber is a reduced toric divisor. A degeneration with these two properties is called a toric degeneration. The name is probably not well-chosen as it suggests a global toric nature, which is not the case as we will emphasize below. A good example to think of is a degeneration of a quartic surface in $\mathbb{P}^{3}$ to the union of the coordinate hyperplanes. More generally, any Calabi-Yau complete intersection in a toric variety has toric degenerations [Gr2]. Thus the notion of toric degeneration is a very versatile one, conjecturally giving all deformation classes of Calabi-Yau varieties with maximally unipotent boundary points.

Our construction has a number of remarkable features. It generalizes the construction of a polarized toric variety from an integral polyhedron (momentum polyhedron)

This work was partially supported by NSF grants 0505325 and 0805328.

(C)2011 International Press 
to non-toric situations in a highly non-trivial, but canonical fashion. It works order by order, each step being controlled by integral affine ("tropical") geometry. This has the striking consequence that any complex geometry feature of the degeneration that is determined on a finite order deformation of the central fibre can, at least in principle, be read off tropically. An important ingredient in this algorithm is the scattering construction, introduced by Kontsevich and Soibelman in a rigid-analytic setup in dimension two [KoSo].

The purpose of these notes is to provide an extended introduction to [GrSi2]. The emphasis is on highlighting some features of the construction by going through examples explicitly. To avoid repeating ourselves, we will introduce most concepts in an ad hoc fashion and refer to $[\mathbf{G r S i 2}]$ for the general case and more technical definitions.

\section{Purely toric constructions}

1.1. Toric varieties from polyhedra. To start with let us recall the algebraicgeometric construction of a toric variety from a convex integral polyhedron $\sigma \subseteq$ $M_{\mathbb{R}}$, the intersection of finitely many closed halfspaces. To keep track of functorial behaviour we work in $M_{\mathbb{R}}:=M \otimes_{\mathbb{Z}} \mathbb{R}$ for some free abelian group $M \simeq \mathbb{Z}^{n}$ of rank $n$. If $\sigma$ is bounded it is the convex hull in $M_{\mathbb{R}}$ of finitely many points in $M \subseteq M_{\mathbb{R}}$. In any case, for each face $\tau \subseteq \sigma$ we have the cone generated by $\sigma$ relative to $\tau$ :

$$
K_{\tau} \sigma:=\mathbb{R}_{\geq 0} \cdot(\sigma-\tau)=\left\{m \in M_{\mathbb{R}} \mid \exists m_{0} \in \tau, m_{1} \in \sigma, \lambda \in \mathbb{R}_{\geq 0}: m=\lambda \cdot\left(m_{1}-m_{0}\right)\right\} .
$$

These cones are finite rational polyhedral, that is, there exist $u_{1}, \ldots, u_{s} \in M$ with

$$
K_{\tau} \sigma=C\left(u_{1}, \ldots, u_{s}\right):=\mathbb{R}_{\geq 0} \cdot u_{1}+\cdots+\mathbb{R}_{\geq 0} \cdot u_{s} .
$$

Note also that $K_{\tau} \sigma \cap\left(-K_{\tau} \sigma\right)=T_{\tau}=\tau-\tau$, the tangent space to $\tau$. So $K_{\tau} \sigma$ is strictly convex if and only if $\tau$ is a vertex.

The integral points of $K_{\tau} \sigma$ define a subring $\mathbb{C}\left[K_{\tau} \sigma \cap M\right]$ of the Laurent polynomial ring $\mathbb{C}[M] \simeq \mathbb{C}\left[z_{1}^{ \pm 1}, \ldots, z_{n}^{ \pm n}\right]$ by restricting the exponents to integral points of $K_{\tau} \sigma$. For $m \in K_{\tau} \sigma \cap M$ we write $z^{m}$ for the corresponding monomial. The invertible elements of this ring are precisely the monomials $c z^{m}$ with $m \in \Lambda_{\tau}:=T_{\tau} \cap M$ and $c \in \mathbb{C} \backslash\{0\}$.

EXAmPle 1.1. Let $\sigma \subseteq \mathbb{R}^{2}$ be the triangle with vertices $v_{1}=(0,0), v_{2}=(1,0)$, $v_{3}=(0, a)$ with $a \in \mathbb{N} \backslash\{0\}$. Then

$$
\begin{aligned}
& K_{v_{1}} \sigma=C((1,0),(0,1)) \\
& K_{v_{2}} \sigma=C((-1, a),(-1,0)) \\
& K_{v_{3}} \sigma=C((0,-1),(1,-a))
\end{aligned}
$$

For $i=1,3$ the monoid of integral points $K_{v_{i}} \sigma \cap \mathbb{Z}^{2} \subseteq \mathbb{Z}^{2}$ is freely generated by the primitive generators $(1,0),(0,1)$ and $(0,-1),(1,-a)$ of the extremal rays. In other words,

$$
\mathbb{N}^{2} \longrightarrow K_{v_{1}} \sigma \cap \mathbb{Z}^{2}, \quad(\alpha, \beta) \longmapsto \alpha \cdot(1,0)+\beta \cdot(0,1)=(\alpha, \beta)
$$


and

$$
\mathbb{N}^{2} \longrightarrow K_{v_{3}} \sigma \cap \mathbb{Z}^{2}, \quad(\alpha, \beta) \longmapsto \alpha \cdot(0,-1)+\beta \cdot(1,-a)=(\beta,-\alpha-\beta a)
$$

are isomorphisms of additive monoids. This shows

$$
\mathbb{C}\left[K_{v_{i}} \sigma \cap \mathbb{Z}^{2}\right] \simeq \mathbb{C}[x, y], \quad i=1,3,
$$

as abstract rings. For $i=2$ the integral generators $(-1, a),(-1,0)$ of the extremal rays of $K_{v_{2}} \sigma$ generate a proper sublattice of $\mathbb{Z}^{2}$ of index $a=\operatorname{det}\left(\begin{array}{cc}-1 & -1 \\ a & 0\end{array}\right)$. Thus $(-1,0),(-1, a)$ also do not suffice to generate $K_{v_{2}} \sigma \cap \mathbb{Z}^{2}$ as a monoid, for $a>1$. It is not hard to show that a minimal set of generators of $K_{v_{2}} \sigma$ rather consists of the $a+1$ elements

$$
(-1,0),(-1,1), \ldots,(-1, a) .
$$

A good way to view $\mathbb{C}\left[K_{v_{2}} \sigma \cap \mathbb{Z}^{2}\right]$ is as the ring of invariants of $\mathbb{C}[x, y]$ under the diagonal action of $\mathbb{Z} / a$ by $a$-th roots of unity $\zeta \in \mathbb{C}, \zeta^{a}=1$ :

$$
x \longmapsto \zeta \cdot x, \quad y \longmapsto \zeta \cdot y .
$$

Under this identification $z^{(-1, i)} \in \mathbb{C}\left[K_{v_{2}} \sigma \cap \mathbb{Z}^{2}\right]$ corresponds to the invariant monomial $x^{i} y^{a-i}$.

The remaining rings associated to higher dimensional faces of $\sigma$ are

$$
\mathbb{C}\left[K_{\tau} \sigma\right] \simeq \begin{cases}\mathbb{C}\left[x, y^{ \pm 1}\right], & \operatorname{dim} \tau=1 \\ \mathbb{C}\left[x^{ \pm 1}, y^{ \pm 1}\right], & \tau=\sigma\end{cases}
$$

As the example indicates, rings of the form $\mathbb{C}\left[K_{\tau} \sigma \cap M\right]$ (toric rings) can be difficult to describe in terms of generators and relations. To obtain examples that can be easily written down in classical projective algebraic geometry, in this paper we therefore almost exclusively restrict ourselves to polyhedra $\sigma$ with $K_{v} \sigma \cap M \simeq \mathbb{N}^{n}$ as a monoid, for any vertex $v \in \sigma$. If $m_{1}, \ldots, m_{s}$ are the generators of the extremal rays of $K_{v} \sigma$, a necessary and sufficient condition for this to be true is $s=n$ and $\operatorname{det}\left(m_{1}, \ldots, m_{s}\right)=1$.

Now given a convex integral polyhedron $\sigma \subseteq M_{\mathbb{R}}$, with $\operatorname{dim} \sigma=n$ for simplicity, and a face $\tau \subseteq \sigma$ we obtain the affine toric variety

$$
U_{\tau}:=\operatorname{Spec}\left(\mathbb{C}\left[K_{\tau} \sigma \cap M\right]\right) .
$$

Since $\mathbb{C}\left[K_{\tau} \sigma \cap M\right] \subseteq \mathbb{C}[M]$ any $U_{\tau}$ contains the algebraic torus $U_{\sigma}=\operatorname{Spec}(\mathbb{C}[M]) \simeq$ $\mathbb{G}_{m}^{n}$. More generally, if $\tau \subseteq \tau^{\prime}$ then $\mathbb{C}\left[K_{\tau} \sigma \cap M\right]$ is canonically a subring of $\mathbb{C}\left[K_{\tau^{\prime}} \sigma \cap M\right]$, and hence we have an open embedding

$$
U_{\tau^{\prime}} \longrightarrow U_{\tau}
$$

These open embeddings are mutually compatible. Hence the $U_{\tau}$ glue to a scheme $X_{\sigma}$ of dimension $\operatorname{dim} \sigma$. In other words, there are open embeddings $U_{\tau} \rightarrow X_{\sigma}$ inducing the morphisms $U_{\tau^{\prime}} \rightarrow U_{\tau}$ for all $\tau \subseteq \tau^{\prime} \subseteq \sigma$. The multiplication action on $U_{\sigma}=\mathbb{G}_{m}^{n}$ extends to $X_{\sigma}$. Hence $X_{\sigma}$ is a toric variety. Note that according to this definition 
toric varieties have a distinguished closed point, the unit of $\mathbb{G}_{m}^{n}$. Moreover, for faces $\tau^{\prime} \subseteq \tau \subseteq \sigma$ the ring epimorphism

$$
\mathbb{C}\left[K_{\tau^{\prime}} \sigma \cap M\right] \longrightarrow \mathbb{C}\left[K_{\tau^{\prime}} \tau \cap M\right], \quad z^{m} \longmapsto \begin{cases}z^{m}, & m \in K_{\tau^{\prime}} \tau \\ 0, & \text { otherwise }\end{cases}
$$

induces a closed embedding $\iota_{\tau}: X_{\tau} \rightarrow X_{\sigma}$ with image disjoint from $\mathbb{G}_{m}^{n} \subseteq X_{\sigma}$ unless $\tau=\sigma$. The images of the various $X_{\tau}$ are called toric strata of $X_{\sigma}$, the image of $U_{\sigma}=\mathbb{G}_{m}^{n}$ the big cell. If $\tau, \tau^{\prime} \subseteq \sigma$ are two faces it holds

$$
\iota_{\tau}\left(X_{\tau}\right) \cap \iota_{\tau^{\prime}}\left(X_{\tau^{\prime}}\right)=\iota_{\tau \cap \tau^{\prime}}\left(X_{\tau \cap \tau^{\prime}}\right) .
$$

Here we make the convention $X_{\emptyset}:=\emptyset$. Hence the face lattice of $\sigma$ readily records the intersection pattern of the toric strata of $X_{\sigma}$. In particular, the facets (codimension one faces) of $\sigma$ are in one-to-one correspondence with the toric prime divisors, the irreducible Weil divisors that are invariant under the torus action.

ExAmple 1.2. For $\sigma=\operatorname{conv}\{(0,0),(1,0),(0, a)\}$ from Example 1.1 we claim that $X_{\sigma}$ is the weighted projective plane $\mathbb{P}(1, a, 1)$. Recall that $\mathbb{P}(1, a, 1)$ is the quotient of $\mathbb{A}^{3} \backslash\{(0,0,0)\}$ by the action of $\mathbb{G}_{m}$ that on closed points is given by

$$
\lambda \cdot\left(x_{0}, x_{1}, x_{2}\right)=\left(\lambda x_{0}, \lambda^{a} x_{1}, \lambda x_{2}\right), \quad \lambda \in \mathbb{C}^{*} .
$$

In fact, on $\mathbb{A}^{3} \backslash V\left(x_{0}\right)$ the ring of invariants of the action is $\mathbb{C}[x, y]$ with $x=x_{1} / x_{0}^{a}$, $y=x_{2} / x_{0}$ and similarly on $\mathbb{A}^{3} \backslash V\left(x_{2}\right)$. On the other hand, on $\mathbb{A}^{3} \backslash V\left(x_{1}\right)$ the ring of invariants is generated by $x_{0}^{i} x_{2}^{a-i} / x_{1}, i=1, \ldots, a$. This is the ring of invariants of the diagonal $\mathbb{Z} / a$-action on $\mathbb{A}^{2}$. Hence $\mathbb{P}(1, a, 1)$ has an affine open covering with spectra of the rings $\mathbb{C}\left[K_{v_{i}} \sigma \cap \mathbb{Z}^{2}\right]$ discussed in Example 1.1. The gluing morphisms between these open sets are the same as given by toric geometry.

By construction the scheme $X_{\sigma}$ depends only on the cones $K_{\tau} \sigma$, hence only on the normal fan of $\sigma$ with elements the dual cones $\left(K_{\tau} \sigma\right)^{\vee} \subseteq M_{\mathbb{R}}^{*}$. More generally, toric varieties are constructed from fans. In particular, integrality and boundedness of $\sigma$ can be weakened to rationality of the cones $K_{\tau} \sigma$. Those toric varieties coming from integral polyhedra (bounded or not) are endowed with a toric ample line bundle. In fact, defining the cone over $\sigma$

$$
C(\sigma):=\operatorname{cl}\left(\mathbb{R}_{\geq 0} \cdot(\sigma \times\{1\})\right) \subseteq M_{\mathbb{R}} \times \mathbb{R},
$$

the ring $\mathbb{C}[C(\sigma) \cap(M \times \mathbb{Z})]$ is graded by $\operatorname{deg} z^{(m, h)}:=h \in \mathbb{N}$. Taking the closure cl here is important in the unbounded case. It adds the asymptotic cone $\lim _{a \rightarrow 0} a \cdot \sigma$ to $M_{\mathbb{R}} \times\{0\}$. It is then not hard to see that one has a canonical isomorphism

$$
X_{\sigma} \simeq \operatorname{Proj}(\mathbb{C}[C(\sigma) \cap(M \times \mathbb{Z})]) .
$$

Although $\mathbb{C}[C(\sigma) \cap(M \times \mathbb{Z})]$ is not in general generated in degree 1, integrality of the vertices of $\sigma$ implies that the sheaf $\mathcal{O}(1)$ on the right-hand side is nevertheless locally free. This yields the toric ample line bundle mentioned above. 
1.2. Toric degenerations of toric varieties. Now let us see how certain unbounded polyhedra naturally lead to toric degenerations with general fibre a toric variety. Let $\tilde{\sigma} \subseteq M_{\mathbb{R}} \times \mathbb{R}$ be an $(n+1)$-dimensional convex integral polyhedron that is closed under positive translations in the last coordinate:

$$
\tilde{\sigma}=\tilde{\sigma}+\left(0 \times \mathbb{R}_{\geq 0}\right) .
$$

Let $q: M_{\mathbb{R}} \times \mathbb{R} \rightarrow M_{\mathbb{R}}$ be the projection and

$$
\sigma:=q(\tilde{\sigma}) .
$$

Then the non-vertical part of $\partial \tilde{\sigma}$ is the graph of a piecewise affine function

$$
\varphi: \sigma \rightarrow \mathbb{R}
$$

with rational slopes. The domains of affine linearity of $\varphi$ define a decomposition $\mathscr{P}$ of $\sigma$ into convex polyhedra. In terms of this data, $\tilde{\sigma}$ is the upper convex hull of the graph of $\varphi$ :

$$
\tilde{\sigma}=\left\{(m, h) \in M_{\mathbb{R}} \times \mathbb{R} \mid h \geq \varphi(m)\right\} .
$$

Thus $\tilde{\sigma}$ is equivalent to a polyhedral decomposition $\mathscr{P}$ of the convex integral polyhedron $\sigma$ together with a function $\varphi$ on $\sigma$ that is piecewise affine and strictly convex with respect to $\mathscr{P}$ and takes integral values at the vertices of $\mathscr{P}$.

Now $X_{\tilde{\sigma}}$ is an $(n+1)$-dimensional toric variety that comes with a toric morphism

$$
\pi: X_{\tilde{\sigma}} \rightarrow \mathbb{A}^{1} .
$$

In fact, each of the rings $\mathbb{C}\left[K_{\tilde{\tau}} \tilde{\sigma} \cap(M \times \mathbb{Z})\right]$ is naturally a $\mathbb{C}[t]$-algebra by letting $t=$ $z^{(0,1)}$, and the gluing morphisms are homomorphisms of $\mathbb{C}[t]$-algebras. The preimage of the closed point $0 \in \mathbb{A}^{1}$ is set-theoretically the union of toric prime divisors of $X_{\tilde{\sigma}}$ that map non-dominantly to $\mathbb{A}^{1}$. It is reduced if and only if $\varphi$ has integral slopes, that is, takes integral values at all integral points, not just the vertices. To see this let $\tilde{v}=(v, \varphi(v)) \in \tilde{\sigma}$ be a vertex and $\varphi_{v}$ a piecewise linear function on $M_{\mathbb{R}}$ which agrees with $\varphi(v+\cdot)-\varphi(v)$ close to 0 . In other words, the graph of $\varphi_{v}$ is the boundary of the tangent cone

$$
K_{\tilde{v}} \tilde{\sigma}=\left\{(m, h) \in M_{\mathbb{R}} \times \mathbb{R} \mid h \geq \varphi_{v}(m)\right\}
$$

of $\tilde{\sigma}$ at $\tilde{v}$. A $\mathbb{C}$-basis for $\mathbb{C}\left[K_{\tilde{v}} \tilde{\sigma} \cap(M \times \mathbb{Z})\right] /(t)$ is given by

$$
z^{(m, h)}, \quad \varphi_{v}(m) \leq h<\varphi_{v}(m)+1,
$$

and $z^{(m, h)}$ is nilpotent modulo $(t)$ if and only if $\varphi_{v}(m)<h$, that is, if $\varphi_{v}(m)$ is not integral.

Assume now that $\varphi(m) \in \mathbb{Z}$ for all $m \in \sigma \cap M$. Then $\mathbb{C}\left[K_{\tilde{v}} \tilde{\sigma} \cap(M \times \mathbb{Z})\right] /(t)$ has one monomial generator $z^{\left(m, \varphi_{v}(m)\right)}$ for any $m \in K_{v} \sigma \cap M$, and the relations are

$$
z^{\left(m, \varphi_{v}(m)\right)} \cdot z^{\left(m^{\prime}, \varphi_{v}\left(m^{\prime}\right)\right)}= \begin{cases}z^{\left(m+m^{\prime}, \varphi_{v}\left(m+m^{\prime}\right)\right)}, & \exists \tau \in \mathscr{P}: v \in \tau \text { and } m, m^{\prime} \in K_{v} \tau \\ 0, & \text { otherwise. }\end{cases}
$$



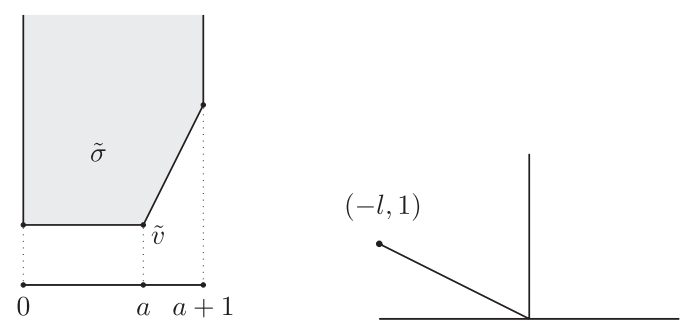

Figure 1.1. The polyhedron $\tilde{\sigma}$ (left) and the normal fan of $\tilde{\sigma}$ (right).

In other words, $\pi^{-1}(0)$ is the scheme-theoretic sum (fibred coproduct) of the $n$-dimensional toric varieties

$$
X_{\tilde{\tau}} \simeq X_{q(\tilde{\tau})}
$$

with $\tilde{\tau} \subset \partial \tilde{\sigma}$ projecting bijectively onto some $\tau \in \mathscr{P}[n]$. These are precisely the toric prime divisors of $X_{\tilde{\sigma}}$ mapping non-dominantly to $\mathbb{A}^{1}$. As in $[\mathbf{G r S i 2}] \mathscr{P}{ }^{[k]}$ denotes the set of $k$-dimensional cells of the polyhedral complex $\mathscr{P}$.

To understand general fibres $\pi^{-1}(t), t \neq 0$, we localize at $t$. This has the effect of removing the lower boundary of $\tilde{\sigma}$, that is, of going over to $\tilde{\sigma}+(0 \times \mathbb{R})=\sigma \times \mathbb{R}$. Thus

$$
\pi^{-1}\left(\mathbb{A}^{1} \backslash\{0\}\right)=X_{\sigma \times \mathbb{R}}=X_{\sigma} \times\left(\mathbb{A}^{1} \backslash\{0\}\right) .
$$

Thus each general fibre $\pi^{-1}(t), t \neq 0$, is canonically isomorphic to $X_{\sigma}$. Note, however, that these isomorphisms degenerate as $t$ approaches 0 .

EXAMPle 1.3. Here is a degeneration of $\mathbb{P}^{1}$ to two copies of $\mathbb{P}^{1}$ featuring an

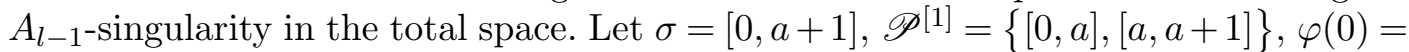
$\varphi(a)=0, \varphi(a+1)=l$ as in Figure 1.1. The slopes of $\varphi$ are 0 and $l$ on the two 1-cells.

The boundary of $\tilde{\sigma}$ has two non-vertical components. Each gives one of the two irreducible components of $\pi^{-1}(0)$. Their point of intersection is the 0 -dimensional toric stratum defined by the vertex $\tilde{v}=(a, 0)$ of $\tilde{\sigma}$. The monoid $K_{\tilde{v}} \tilde{\sigma} \cap \mathbb{Z}^{2}$ has generators $m_{1}=(-1,0), m_{2}=(1, l), m_{3}=(0,1)$ with relation $m_{1}+m_{2}=l \cdot m_{3}$. Hence,

$$
\mathbb{C}\left[K_{\tilde{v}} \tilde{\sigma} \cap \mathbb{Z}^{2}\right] \simeq \mathbb{C}\left[z_{1}, z_{2}, t\right] /\left(z_{1} z_{2}-t^{l}\right),
$$

with $z_{i}=z^{m_{i}}$ for $i=1,2$ and $t=z^{(0,1)}$ defining $\pi$. This is a local model of a smoothing of a nodal singularity with an $A_{l-1}$-singularity in the total space. Thus the changes of slope of $\varphi$ at the non-maximal cells of $\mathscr{P}$ determine the singularities of the total space.

Another way to understand the total space is from the normal fan of $\tilde{\sigma}$. It can be obtained by subdividing the fan of $\mathbb{A}^{1} \times \mathbb{P}^{1}$ by the ray through $(-l, 1)$. This corresponds to a weighted blow up at one of the zero-dimensional toric strata, leading to another $\mathbb{P}^{1}$ over $0 \in \mathbb{A}^{1}$ and the $A_{l-1}$-singularity.

Note also that the length $a$ of the interval is completely irrelevant to the complex geometry; it only changes the polarization which we did not care about at this point. 
EXAmPLE 1.4. For a two-dimensional example consider the convex hull

$$
\sigma=\operatorname{conv}\{(1,0),(0,1),(-1,0),(0,-1)\}
$$

with the polyhedral decomposition into 4 standard 2-simplices shown in the figure below. At $(x, y) \in \sigma$ the function $\varphi$ is given by $0, x, y$ and $x+y$, respectively, depending on the maximal cell containing $(x, y)$ as shown in the figure. Thus the slope of $\varphi$ changes by 1 along each interior 1-cell of $\mathscr{P}$.

The associated degeneration has as central fibre a union of 4 copies of $\mathbb{P}^{2}$, glued pairwise along the coordinate lines as indicated by $\mathscr{P}$. Thus the singular locus is a union of 4 projective lines, joined in one point $X_{\tilde{v}}$, where $\tilde{v}=(0,0,0) \in \tilde{\sigma}$. The monoid $K_{\tilde{v}} \tilde{\sigma}$ is generated by $m_{1}=(1,0,1), m_{2}=(0,1,1), m_{3}=(-1,0,0), m_{4}=(0,-1,0)$ fulfilling the single relation $m_{1}+m_{3}=m_{2}+m_{4}$. Thus

$$
\mathbb{C}\left[K_{\tilde{v}} \tilde{\sigma} \cap \mathbb{Z}^{3}\right] \simeq \mathbb{C}\left[z_{1}, z_{2}, z_{3}, z_{4}\right] /\left(z_{1} z_{3}-z_{2} z_{4}\right),
$$

with $\mathbb{C}[t]$-algebra structure defined by $t=z^{(0,0,1)}=z_{1} z_{3}=z_{2} z_{4}$. This shows that the total space $X_{\tilde{\sigma}}$ has a singular point isomorphic to the origin in the affine cone over a smooth quadric, while the central fibre is a product of two normal crossing singularities.

The general fibre is isomorphic to $X_{\sigma}$, as always, which here is a toric $\mathbb{Z} / 2$-quotient of $\mathbb{P}^{1} \times \mathbb{P}^{1}$. In fact, $\left(\begin{array}{rr}1 & 1 \\ -1 & 1\end{array}\right)$ maps the fan of $\mathbb{P}^{1} \times \mathbb{P}^{1}$ to the normal fan of $\sigma$. Restricted to the big cell $\mathbb{G}_{m}^{2} \subset \mathbb{P}^{1} \times \mathbb{P}^{1}$, this map is given by

$$
\mathbb{C}\left[u^{ \pm 1}, v^{ \pm 1}\right] \longrightarrow \mathbb{C}\left[x^{ \pm 1}, y^{ \pm 1}\right], \quad u \longmapsto x y, v \longmapsto x^{-1} y .
$$

The subring of $\mathbb{C}\left[x^{ \pm 1}, y^{ \pm 1}\right]$ generated by $x^{ \pm 1} y^{ \pm 1}$ is the invariant ring for the involution $(x, y) \rightarrow(-x,-y)$, and this involution extends to $\mathbb{P}^{1} \times \mathbb{P}^{1}$. Note that $X_{\sigma}$ has 4 isolated quotient singularities. These

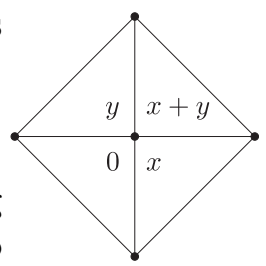
correspond to the vertices of $\sigma$.

It is also instructive to write down this degeneration embedded projectively. As explained at the end of $\S 1.1$ we have to take the integral points of the cone $C(\tilde{\sigma}) \subseteq \mathbb{R}^{4}$ over $\tilde{\sigma}$ as generators of a graded $\mathbb{C}[t]$-algebra, with the degree given by the projection to the last coordinate and $t=z^{(0,0,1,0)}$. Now $\mathbb{C}\left[C(\tilde{\sigma}) \cap \mathbb{Z}^{4}\right]$ is generated as a $\mathbb{C}[t]$-algebra by the monomials

$$
X=z^{(1,0,1,1)}, Y=z^{(-1,0,0,1)}, Z=z^{(0,1,1,1)}, W=z^{(0,-1,0,1)}, U=z^{(0,0,0,1)} .
$$

Note that these generators are in one-to-one correspondence with the integral points of $\sigma$. The relations are

$$
X Y-t U^{2}, \quad Z W-t U^{2}
$$

This exhibits $X_{\tilde{\sigma}}$ as the intersection of two quadrics in $\mathbb{P}_{\mathbb{A}^{1}}^{4}=\mathbb{A}^{1} \times \mathbb{P}^{4}$. 


\section{Introducing singular affine structures}

From a birational classification point of view toric varieties are boring as they are all rational. In particular, it is impossible to construct degenerations of non-rational varieties directly by the method of $\S 1.2$. The idea in $[\mathbf{G r S i 2}]$ is that one can get a much larger and more interesting class of degenerations by gluing toric pieces in a non-toric fashion. The central fibre is still represented by a cell complex $\mathscr{P}$ of integral polyhedra, but the integral affine embedding of the cell complex into $\mathbb{R}^{n}$ exists only locally near each vertex. In other words, the underlying topological space of $\mathscr{P}$ is an integral affine manifold $B$, with singularities on a cell complex $\Delta \subseteq B$ of real codimension 2 that is a retract of $\left|\mathscr{P}^{[n-1]}\right| \backslash\left|\mathscr{P}^{[0]}\right|$. We then construct the total space $\mathfrak{X}$ of the degeneration order by order, by gluing torically constructed non-reduced varieties, "thickenings" of toric varieties so to speak, in a non-toric fashion.

Thus our starting data are integral cell-complexes with compatible integral affine charts near the vertices. We call these integral tropical manifolds ([GrSi2], Definition 1.2) because they arise naturally as the bounded parts of the embedded tropical varieties associated to the degeneration.

2.1. Degenerations of hypersurfaces. A hypersurface $X \subseteq \mathbb{P}^{n+1}$ of degree $d \leq n+2$ can be degenerated to a union of $d$ coordinate hyperplanes simply by deforming the defining equation. For example, for $n=2$ let $f \in \mathbb{C}\left[X_{0}, \ldots, X_{3}\right]$ be a general homogeneous polynomial of degree $d \leq 4$. Then

$$
X_{0} \ldots X_{d-1}+t f=0
$$

defines a family $\pi: Y \rightarrow \mathbb{A}^{1}$ with $\pi^{-1}(0)$ a union of $d$ coordinate hyperplanes. This is not a semistable family because $Y$ is not smooth at the intersection of $V(f)$ with the singular locus of $V\left(X_{0} \cdot \ldots \cdot X_{d-1}\right)$. The latter consists of $\left(\begin{array}{l}d \\ 2\end{array}\right)$ projective lines $V\left(X_{i}, X_{j}\right), 0 \leq i<j \leq d-1$. Since $f$ is general the intersection of $V(f)$ with any of these projective lines consists of $d$ reduced points with two nonzero coordinate entries each, that is, not equal to the 4 special points $[1,0,0,0],[0,1,0,0],[0,0,1,0],[0,0,0,1]$. Near any of these points $Y \rightarrow \mathbb{A}^{1}$ is locally analytically given by the projection of the three-dimensional $A_{1}$-singularity

$$
V(x y-w t) \subseteq \mathbb{A}^{4}
$$

to the $t$-coordinate. Note that for $w \neq 0$ this is a product of a semistable degeneration of a curve with $\mathbb{A}^{1} \backslash\{0\}$, but this fails at $w=0$, which contains the singular point of $Y$.

While the local model of this degeneration is still toric, the singular points of $Y$ are general points of one-dimensional toric strata of $\pi^{-1}(0)$. Hence this is a very different degeneration than the torically constructed ones in $§ 1.2$. Our first aim is to obtain this local degeneration naturally from a tropical manifold. This was the starting point for [GrSi2] in March 2004.

2.2. A singular affine manifold. There is a famous two-dimensional singular integral affine manifold in the theory of integrable systems, called the focus-focus 

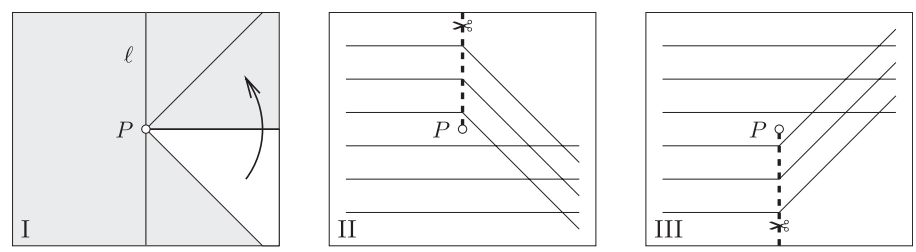

Figure 2.1. Various charts I-III for the focus-focus singularity: On the left, the shaded area is a fundamental domain for $B$. In the two other figures, the dashed vertical lines indicate the parts of $\ell$ to be removed; the horizontal curves are all straight affine lines.

singularity $[\mathbf{W i}$ ], which is the model for our singular affine manifolds $B$ at general points of $\Delta$, the codimension 2 singular locus of the affine structure. An (integral) affine structure on a topological manifold is an atlas with transition functions in the (integral) affine linear group. Parallel transport of tangent vectors is well-defined on such manifolds. In fact, an affine manifold comes naturally with a flat, torsion-free, but usually non-metric connection. The focus-focus singularity is (the germ at the origin $P$ of) $\mathbb{R}^{2}$ with an integral affine structure away from $P$ such that parallel transport counterclockwise around $P$ gives the transformation $\left(\begin{array}{ll}1 & 0 \\ 1 & 1\end{array}\right)$. This affine manifold $B$ can be constructed by gluing $\mathbb{R}^{2} \backslash\left(\mathbb{R}_{\geq 0} \times\{0\}\right)$ with the given affine structure to itself by the integral affine transformation

$$
(x, y) \longmapsto(x, x+y), \quad \text { for } x \geq 0,-x<y<0,
$$

see Chart I in Figure 2.1. Note that $\partial_{y}$ is an invariant tangent vector, and indeed the projection $(x, y) \rightarrow x$ on $\mathbb{R}^{2} \backslash\left(\mathbb{R}_{\geq 0} \times\{0\}\right)$ to the first coordinate descends to a continuous map $B \rightarrow \mathbb{R}$ that is integral affine away from $P \in B$. The preimage of the origin is a line $\ell$ through $P$.

Alternatively, $B$ can be constructed from the two charts $\mathbb{R}^{2} \backslash\left(\{0\} \times \mathbb{R}_{\geq 0}\right)$ and $\mathbb{R}^{2} \backslash\left(\{0\} \times \mathbb{R}_{\leq 0}\right)$, each covering $B$ minus one half of $\ell$, via

$$
(\mathbb{R} \backslash\{0\}) \times \mathbb{R} \longrightarrow(\mathbb{R} \backslash\{0\}) \times \mathbb{R}, \quad(x, y) \longmapsto \begin{cases}(x, y), & x<0 \\ (x, x+y), & x>0,\end{cases}
$$

see Charts II and III in Figure 2.1. Note that we do not know how to continue any non-vertical affine line across $P$.

2.3. The basic example. The focus-focus singularity admits a polyhedral decomposition by decomposing along the invariant line $\ell$. This decomposition has two maximal cells $\sigma_{ \pm}$, with preimages $\mathbb{R}_{\geq 0} \times \mathbb{R}$ and $\mathbb{R}_{\leq 0} \times \mathbb{R}$ in any of the charts of Figure 2.1. We can also define a strictly convex, integral affine function $\varphi: B \rightarrow \mathbb{R}$ by letting $\varphi=0$ on the left maximal cell $\sigma_{-}$and $\varphi(x, y)=x$ on the right maximal cell $\sigma_{+}$. Again, this is independent of the chosen chart, and it takes integral values at any integral point of $B$. 
Now let us try to treat this situation with a singularity in the same way as we did in $\S 1.2$. We can still describe the central fibre as before:

$$
\begin{aligned}
X_{0} & =X_{\sigma_{-}} \amalg_{X_{\rho}} X_{\sigma_{+}}, \\
X_{\sigma_{-}} & =\operatorname{Spec}\left(\mathbb{C}\left[x, w^{ \pm 1}\right]\right), X_{\sigma_{+}}=\operatorname{Spec}\left(\mathbb{C}\left[y, w^{ \pm 1}\right]\right), X_{\rho}=\operatorname{Spec}\left(\mathbb{C}\left[w^{ \pm 1}\right]\right),
\end{aligned}
$$

where $\rho=\sigma_{-} \cap \sigma_{+}, x=z^{(-1,0)}, y=z^{(1,0)}, w=z^{(0,1)}$. By writing this it is important to fix one affine realization of $\sigma_{ \pm}$, say the Chart II in Figure 2.1. Intrinsically, the exponent $(-1,0)$ defining $x$ is an integral tangent vector on $\sigma_{-}$, while the exponent $(1,0)$ defining $y$ is an integral tangent vector on $\sigma_{+}$. For $w$ we can take either maximal cell, because the exponent $(0,1)$ just represents the global integral vector field $\partial_{y}$ defined above.

Continuing to work in Chart II the procedure of $\$ 1.2$ yields the deformation $V(x y-t)$ of $V(x y) \subseteq \mathbb{A}^{2} \times\left(\mathbb{A}^{1} \backslash\{0\}\right)$. But in Chart III the tangent vector $(1,0)$ of $\sigma_{+}$changes to $(1,1)$. Since $z^{(1,1)}=w y$ the same procedure applied in this chart therefore yields $V(x y-w t)$ for the deformation. Because $w$ is invertible $V(x y-t)$ and $V(x y-w t)$ are isomorphic as schemes over $\mathbb{C}[t]$, but not as deformation with the given embedding of $X_{0}$. Of course, this phenomenon is just due to the affine monodromy around $P$. As expected, the singularities of the affine structure lead to inconsistencies in the naive approach using toric geometry.

The starting point to overcome this problem is to work modulo $t^{k+1}$. In other words, we want to construct a $k$-th order deformation $X_{k} \rightarrow \operatorname{Spec}\left(\mathbb{C}[t] /\left(t^{k+1}\right)\right)$. The topological spaces of $X_{0}$ and $X_{k}$ are the same, so we only have to deal with the structure sheaf. We now use toric geometry merely to define the correct non-reduced versions ( $k$-th order thickenings) of the toric strata. We then glue two maximaldimensional strata intersecting in codimension one by a non-toric automorphism of the common codimension one stratum, but the choice of this automorphism is different depending on which chart we use.

In the present example, the thickenings of the toric strata suggested by toric geometry are given by the rings

$$
\begin{aligned}
R_{\sigma_{-}}^{k} & =S_{k}\left[x_{1}, y_{1}, w^{ \pm 1}\right] /\left(x_{1} y_{1}-t, y_{1}^{k+1}\right), \\
R_{\sigma_{+}}^{k} & =S_{k}\left[x_{2}, y_{2}, w^{ \pm 1}\right] /\left(x_{2} y_{2}-t, x_{2}^{k+1}\right), \\
R_{\rho, \sigma_{-}}^{k} & =S_{k}\left[x_{1}, y_{1}, w^{ \pm 1}\right] /\left(x_{1} y_{1}-t, x_{1}^{k+1}, y_{1}^{k+1}\right), \\
R_{\rho, \sigma_{+}}^{k} & =S_{k}\left[x_{2}, y_{2}, w^{ \pm 1}\right] /\left(x_{2} y_{2}-t, x_{2}^{k+1}, y_{2}^{k+1}\right),
\end{aligned}
$$

with $S_{k}=\mathbb{C}[t] /\left(t^{k+1}\right)$. Here we distinguish between $x$ and $y$ as monomials on $\sigma_{-}$and on $\sigma_{+}$. Thus $y_{1}=z^{(1,0,1)}$ in either chart, while $y_{2}=z^{(1,0,1)}$ in Chart II and $y_{2}=z^{(1,1,1)}$ in Chart III. These monomials only depend on the affine structure on $\sigma_{ \pm}$and hence have an intrinsic meaning. For the thickening of the $\rho$-stratum, however, we obtain two rings, depending on which maximal cell $\sigma_{ \pm}$the monomials live on. For the monomial $w$ we don't need to make any choices because it corresponds to a globally defined vector field. In any case, we have two natural $S_{k}$-algebra epimorphisms

$$
q_{-}: R_{\sigma_{-}}^{k} \longrightarrow R_{\rho, \sigma_{-}}^{k}, \quad q_{+}: R_{\sigma_{+}}^{k} \longrightarrow R_{\rho, \sigma_{+}}^{k},
$$


exhibiting the thickened $\rho$-stratum as closed subscheme of the $\sigma_{--}$and $\sigma_{+}$-stratum, respectively.

Affine geometry suggests two isomorphisms $h_{\mathrm{II}}, h_{\mathrm{III}}: R_{\rho, \sigma_{-}}^{k} \rightarrow R_{\rho, \sigma_{+}}^{k}$, depending on which chart we use to go from $\sigma_{-}$to $\sigma_{+}$:

$$
\begin{array}{cl}
h_{\mathrm{II}}: x_{1} \longmapsto x_{2}, \quad y_{1} \longmapsto y_{2}, & w \longmapsto w \\
h_{\mathrm{III}}: x_{1} \longmapsto w x_{2}, & y_{1} \longmapsto w^{-1} y_{2}, \quad w \longmapsto w
\end{array}
$$

Now comes the point: To remedy the inconsistency caused by this, let $\alpha \in \mathbb{C}^{*}$ and compose $h_{\mathrm{II}}$ with the automorphism

$$
g_{\mathrm{II}}: x_{2} \longmapsto(\alpha+w) \cdot x_{2}, \quad y_{2} \longmapsto(\alpha+w)^{-1} y_{2}, \quad w \longmapsto w
$$

of the localization $\left(R_{\rho, \sigma_{+}}^{k}\right)_{\alpha+w}$. For Chart III we compose with

$$
g_{\mathrm{III}}: x_{2} \longmapsto\left(1+\alpha w^{-1}\right) \cdot x_{2}, \quad y_{2} \longmapsto\left(1+\alpha w^{-1}\right)^{-1} y_{2}, \quad w \longmapsto w .
$$

We then indeed obtain

$$
\begin{aligned}
& \left(g_{\mathrm{II}} \circ h_{\mathrm{II}}\right)\left(x_{1}\right)=(\alpha+w) \cdot x_{2}=\left(1+\alpha w^{-1}\right) \cdot w x_{2}=\left(g_{\mathrm{III}} \circ h_{\mathrm{III}}\right)\left(x_{1}\right), \\
& \left(g_{\mathrm{II}} \circ h_{\mathrm{II}}\right)\left(y_{1}\right)=(\alpha+w)^{-1} \cdot y_{2}=\left(1+\alpha w^{-1}\right)^{-1} \cdot w^{-1} y_{2}=\left(g_{\mathrm{III}} \circ h_{\mathrm{III}}\right)\left(y_{1}\right) .
\end{aligned}
$$

The result of gluing Spec $R_{\sigma_{-}}^{k}$ and Spec $R_{\sigma_{+}}^{k}$ along the codimension one strata via this isomorphism is given by the fibre product $R_{\sigma_{-}}^{k} \times_{\left(R_{\rho, \sigma_{+}}^{k}\right)_{\alpha+w}} R_{\sigma_{+}}^{k}$. In this fibre product the homomorphism $R_{\sigma_{+}}^{k} \rightarrow\left(R_{\rho, \sigma_{+}}^{k}\right)_{\alpha+w}$ is the composition of $q_{+}$with localization, while $R_{\sigma_{-}}^{k} \rightarrow\left(R_{\rho, \sigma_{+}}^{k}\right)_{\alpha+w}$ composes $q_{-}$and the localization homomorphism with $g_{\mathrm{II}} \circ$ $h_{\mathrm{II}}=g_{\mathrm{III}} \circ h_{\mathrm{III}}$. It can be shown $([\mathbf{G r S i 2}]$, Lemma 2.34) that generators for this fibre product as an $S_{k}\left[w^{ \pm 1}\right]$-algebra are

$$
x=\left(x_{1},(\alpha+w) x_{2}\right), \quad y=\left((\alpha+w) y_{1}, y_{2}\right)
$$

with the single relation (coming from $x_{1} y_{1}=t=x_{2} y_{2}$ )

$$
x y-(\alpha+w) t=0 .
$$

This is the $k$-th order neighbourhood of the surface degeneration discussed in $\S 2.1$, with the $A_{1}$-singularity at $x=y=0$ and $w=-\alpha$.

2.4. General treatment in codimension one. The discussion in $\S 2.3$ generalizes to arbitrary dimension $n$ as follows. Let $\rho$ be a codimension one cell of $\mathscr{P}$, separating the maximal cells $\sigma_{-}, \sigma_{+}$. This gives three toric varieties $X_{\sigma_{ \pm}}$and $X_{\rho}$ with the respective big cells $\operatorname{Spec}\left(\mathbb{C}\left[\Lambda_{\sigma_{ \pm}}\right]\right) \simeq \mathbb{G}_{m}^{n}$ and $\operatorname{Spec}\left(\mathbb{C}\left[\Lambda_{\rho}\right]\right) \simeq \mathbb{G}_{m}^{n-1}$. For an integral polyhedron $\tau$ we use the notation $\Lambda_{\tau}$ for the lattice of integral tangent vector fields on $\tau$. The convex integral piecewise affine function $\varphi$ changes slope along $\rho$ by some integer $k>0$. We then obtain the same rings and gluing morphisms as in $\S 2.3$, except that $w$ has to be replaced by $n-1$ coordinates $w_{1}, \ldots, w_{n-1}$ for $\mathbb{G}_{m}^{n-1} \subseteq X_{\rho}$. 
There are affine charts on the interiors of $\sigma_{ \pm}$and near each vertex of $\rho$. Each vertex $v \in \rho$ suggests an isomorphism $h_{v}: R_{\rho, \sigma_{-}}^{k} \rightarrow R_{\rho, \sigma_{+}}^{k}$. For a different vertex $v^{\prime} \in \rho$ we have $h_{v^{\prime}}\left(z^{m}\right)=h_{v}\left(z^{m}\right)$ for $m \in \Lambda_{\rho}$ because these tangent vectors are globally defined on $\sigma_{-} \cup \sigma_{+}$. But for general $m \in \Lambda_{\sigma_{-}}$, parallel transport $\Pi_{v^{\prime}}$ to $\sigma_{+}$through $v^{\prime}$ is related to parallel transport $\Pi_{v}$ through $v$ as follows:

$$
\Pi_{v^{\prime}}(m)=\Pi_{v}(m)+\left\langle m, \check{d}_{\rho}\right\rangle \cdot m_{v^{\prime} v^{\prime}}^{\rho} .
$$

Here $m_{v^{\prime} v}^{\rho}$ is some element of $\Lambda_{\rho}$, viewed canonically as a subset of $\Lambda_{\sigma_{+}}$, and $\check{d}_{\rho}$ is the primitive generator of $\left(\Lambda_{v^{\prime}} / \Lambda_{\rho}\right)^{*} \simeq \mathbb{Z}$ that evaluates positively on tangent vectors pointing from $\rho$ into $\sigma_{+}$. This monodromy formula implies

$$
h_{v^{\prime}}\left(z^{m}\right)=\left(z^{m_{v^{\prime} v}^{\rho}}\right)^{\left\langle m, \check{d}_{\rho}\right\rangle} \cdot h_{v}\left(z^{m}\right) .
$$

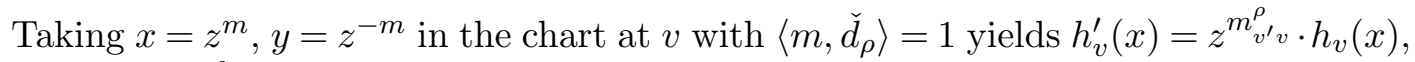
$h_{v}^{\prime}(y)=z^{-m_{v^{\prime} v}^{\rho}} \cdot h_{v}(y)$. Thus to define the gluing invariantly we can compose $h_{v}$ with an automorphism of $R_{\rho, \sigma_{+}}^{k}$ of the form

$$
g_{v}: z^{m} \longmapsto f_{\rho, v}^{-\left\langle\check{d}_{\rho}, m\right\rangle} \cdot z^{m}
$$

for functions $f_{\rho, v} \in \mathbb{C}\left[\Lambda_{\rho}\right]$, indexed by vertices $v$ of $\rho$ and fulfilling the change of vertex formula

$$
f_{\rho, v^{\prime}}=z^{m_{v^{\prime} v}^{\rho}} \cdot f_{\rho, v} .
$$

In the example in $\S 2.3$ we can think of $v$ as lying on the upper half of $\ell, v^{\prime}$ on the lower half and $f_{\rho, v}=1+\alpha w^{-1}, f_{\rho, v^{\prime}}=\alpha+w$. Then $m_{v^{\prime} v}^{\rho}=(0,1), z^{m_{v^{\prime} v}^{\rho}}=w$, and the change of vertex formula reads $\alpha+w=w \cdot\left(1+\alpha w^{-1}\right)$. In the general case the result of gluing is the hypersurface

$$
x y-f_{\rho, v}\left(w_{1}, \ldots, w_{n-1}\right) t^{k}=0 .
$$

\section{Examples without scattering}

In Section 2 we worked in an affine (in the algebraic geometry sense) neighbourhood of a singular point of the total space of the degeneration. We now want to look at projective examples in two dimensions, with one or several singular points. To this end we work with bounded tropical manifolds and use the toric procedure from $\S 1.2$ for the treatment away from the singularities.

3.1. Overview of the general procedure. To obtain degenerations of complete varieties we need to start from a bounded tropical manifold $(B, \mathscr{P})$, where $B$ denotes the underlying singular integral affine manifold and $\mathscr{P}$ is the decomposition into integral polyhedra, together with an (in general multi-valued) integral piecewise affine function $\varphi$. The tropical manifold determines readily the prospective central fibre $X_{0}=\bigcup_{\sigma \in \mathscr{P}_{\max }} X_{\sigma}$ from the maximal cells, glued pairwise torically in codimension one. The most general approach also allows one to compose the gluing 
in codimension one with a toric automorphism, but as this is a rather straightforward generalization that only complicates the formulas we do not include it here. In the notation of $\left[\mathbf{G r S i 2}\right.$ ] this means taking $s_{e}=1$ for any inclusion $e: \omega \rightarrow \tau$ of cells $\omega, \tau \in \mathscr{P}$.

To obtain local models near the singular points we need to choose, for each $\rho \in$ $\mathscr{P}^{[n-1]}$ and vertex $v \in \rho$, a regular function $f_{\rho, v}$ on an open set in $X_{\rho} \subseteq X_{0}$. The open set is the affine neighbourhood

$$
U_{v}:=X_{0} \backslash \bigcup_{\tau \in \mathscr{P}, v \notin \tau} X_{\tau}
$$

of the corresponding point $X_{v} \in X_{0}$. As explained in $\S 2.4$, consistency of the gluing dictates the change of vertex formula (2.3) that relates $f_{\rho, v}$ and $f_{\rho, v^{\prime}}$ for vertices $v, v^{\prime} \in \rho$. There is also a compatibility condition for the $f_{\rho, v}$ for fixed $v$ and all $\rho$ containing a codimension- 2 cell that makes sure everything stays toric étale locally at general points of the toric strata. We will explain this condition when we need it later on (see the discussion after Example 4.1).

Next, the piecewise affine function $\varphi$ determines local toric models for the degeneration, defined by the toric procedure from $\S 1.2$. Explicitly, if $v \in \mathscr{P}$ is a vertex and $\varphi_{v}$ is a piecewise linear representative of $\varphi$ introduced in $\S 1.2$ then the local model near the zero-dimensional toric stratum $X_{v}$ is

$$
\operatorname{Spec}(\mathbb{C}[P]) \longrightarrow \operatorname{Spec}(\mathbb{C}[t]), \quad P=\left\{(m, h) \in M \times \mathbb{Z} \mid h \geq \varphi_{v}(m)\right\} .
$$

Here $t=z^{(0,1)}$ makes $\mathbb{C}[P]$ into a $\mathbb{C}[t]$-algebra. Following the discussion in Section 2 we now work to some finite $t$-order $k$, that is modulo $t^{k+1}$, and decompose according to toric strata. This gives toric local models that depend on $k$, a toric affine open neighbourhood $U_{\omega} \subseteq X_{0}$ of $\operatorname{Int}\left(X_{\omega}\right)$, a toric stratum $X_{\tau}$ intersecting $U_{\omega}$ and a maximal-dimensional reference cell $\sigma \supseteq \tau$. In analogy with $U_{v}$ for $\omega \in \mathscr{P}$ we take

$$
U_{\omega}:=X_{0} \backslash \bigcup_{\tau \in \mathscr{P}, \omega \nsubseteq \tau} X_{\tau} .
$$

Thus we need $\sigma \in \mathscr{P}_{\max }$ and cells $\omega, \tau \in \mathscr{P}$ with $\omega \subseteq \tau \subseteq \sigma$. The last condition is equivalent to $U_{\omega} \cap X_{\tau} \neq \emptyset$. The corresponding ring, localized at all the gluing functions $f_{\rho, v}$ with $\rho \supseteq \tau$, is denoted $R_{\omega \rightarrow \tau, \sigma}^{k}$, see [GrSi2], $\S 2.1$ for details. Thus $\operatorname{Spec} R_{\omega \rightarrow \tau, \sigma}^{k}$ is a $k$-th order non-reduced version ("thickening") of $\left(X_{\tau} \cap U_{\omega}\right) \backslash \bigcup_{\rho \supseteq \tau} V\left(f_{\rho, v}\right), v \in \omega$ arbitrary. Note that for $v, v^{\prime} \in \tau$ the gluing functions $f_{\rho, v}$ and $f_{\rho, v^{\prime}}$ differ by a monomial that is invertible on $U_{\omega}$, so $V\left(f_{\rho, v^{\prime}}\right) \cap U_{\omega}=V\left(f_{\rho, v}\right) \cap U_{\omega}$.

The relation ideal defining $R_{\omega \rightarrow \tau, \sigma}^{k}$ in (the localization at $\prod_{\rho \supseteq \tau} f_{\rho, v}$ of) $\mathbb{C}[P]$ is generated by all monomials $z^{(m, h)},(m, h) \in \Lambda_{\tau} \oplus \mathbb{Z}$, that have $\tau$-order at least $k+1$. Here one defines the $\tau$-order as the maximum, over all $\sigma^{\prime} \in \mathscr{P}_{\max }$ containing $\tau$, of the order of vanishing of $z^{(m, h)}$ on the big cell of $X_{\sigma^{\prime}}$, viewed canonically as a subset of $\operatorname{Spec}(\mathbb{C}[P])$.

Now even in examples with few cells this procedure requires the gluing of many affine schemes. We can, however, sometimes use the following shortcut. The integral points of $B$ provide a basis of sections of a natural very ample line bundle on the 
central fibre $X_{0}$, hence providing a closed embedding $X_{0} \rightarrow \mathbb{P}^{N-1}, N$ the number of integral points of $B$. In sufficiently simple examples the gluing morphisms defining the deformation of $X_{0}$ readily homogenize to describe the $k$-th order deformation of $X_{0}$ as a subspace of $\mathbb{P}_{O_{k}}^{N}, O_{k}=\operatorname{Spec} S_{k}, S_{k}=\mathbb{C}[t] /\left(t^{k+1}\right)$. The toric nature of this construction makes sure that this procedure gives the right local models. This is how we compute most of the examples in this paper.

3.2. One singular point I: Two triangles. For our first series of examples we fit the basic example from $\S 2.3$ into a projective degeneration. This amounts to finding a polyhedral decomposition of a bounded neighbourhood of the singular point $P$ of the focus-focus singularity in Figure 2.1. One of the easiest ways to do this is to take two standard triangles (integrally affine isomorphic to conv $\{(0,0),(1,0),(0,1)\}$ ), one on each half-plane $\sigma_{ \pm}$, and with $P$ at the center of the common edge. In particular, $P$ should not be integral, but rather half-integral. Thus let us first shift our Charts I-III by $(0,1 / 2)$ so that the singular point $P$ is at $(0,1 / 2)$. Now in Chart II take

$$
\sigma_{1}=\operatorname{conv}\{(0,0),(-1,0),(0,1)\}, \quad \sigma_{2}=\operatorname{conv}\{(0,0),(1,0),(0,1)\}
$$

as maximal cells for the polyhedral decomposition, see Figure 3.1. This is one of the two choices making the boundary locally convex. In fact, we can always bring $\sigma_{1}$ to the suggested form by applying a transvection $\left(\begin{array}{ll}1 & 0 \\ a & 1\end{array}\right)$ on Chart I for some $a \in$ $\mathbb{Z}$. This descends to an integral affine transformation of the focus-focus singularity. Then $\sigma_{2}=\operatorname{conv}\{(0,0),(1, b),(0,1)\}$ for some $b \in \mathbb{Z}$. Convexity at $(0,0)$ implies $b \geq 0$, while convexity at $(0,1)$ implies $b \leq 1$ (check in Chart III). Local convexity of $\partial B$ is indispensible to define local toric models for the deformation. Both choices are abstractly isomorphic, $b=0$ making the lower boundary a line, $b=1$ the upper one.

There are 4 integral points on this tropical manifold $B_{1}$, the vertices of $\mathscr{P}$. We interpret these as homogeneous coordinates in a $\mathbb{P}^{3}=\operatorname{Proj}(\mathbb{C}[X, Y, Z, W])$. The central fibre $X_{0}$ is then given by the hypersurface $X Y=0$, because $X$ and $Y$ are the only vertices not contained in the same cell. In fact, if $\mathscr{P}$ consists of standard simplices, $X_{0}$ can be defined by the Stanley-Reisner ideal $[\mathbf{H o}],[\mathbf{S t}]$ of the simplicial complex given by $\mathscr{P}$. This is clear from the definitions.

Now the gluing computation in $\S 2.3$ readily homogenizes. In fact, viewing the computation in Chart II as being homogenized with respect to Z, the relation (2.1) becomes

$$
\frac{X}{Z} \frac{Y}{Z}-t\left(\alpha+\frac{W}{Z}\right)=0
$$
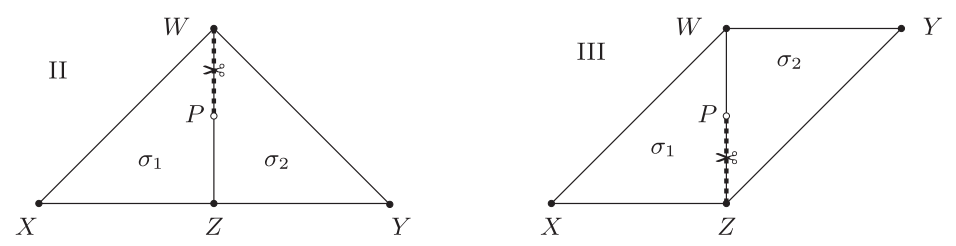

FiguRE 3.1. The two charts defining the tropical manifold $B_{1}$. 


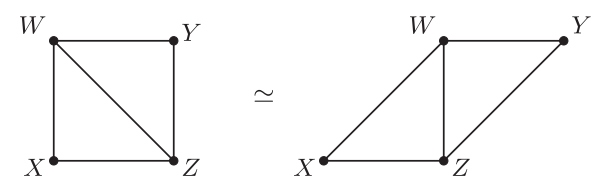

Figure 3.2. Torically constructed degeneration of $\mathbb{P}^{1} \times \mathbb{P}^{1}$

Clearing denominators yields the family of quadric hypersurfaces

$$
X Y-t Z(\alpha Z+W)=0
$$

The analogous computation in the other chart gives the same result. Hence we obtain a projective degeneration with general fibre isomorphic to $\mathbb{P}^{1} \times \mathbb{P}^{1}$. The total space has another $A_{1}$-singularity of a toric nature at $X=Y=Z=0$. This singularity is due to the fact that $\partial B_{1}$ is not straight at $(0,1)$.

It is interesting to compare this deformation to the torically constructed one for a diagonally subdivided unit square with $\varphi$ changing slope by 1 along the diagonal. As shown in Figure 3.2 we again have 4 homogeneous generators $X, Y, Z, W$ and the relation

$$
X Y-t Z W=0
$$

The change of coordinates $W \mapsto \alpha Z+W$ shows that this family is isomorphic to the previous one, the only difference being that now both $A_{1}$-singularities of the total space are at 0 -dimensional toric strata of $\mathbb{P}^{3}$. Thus in a sense, by introducing the singular point in the interior we have straightened the boundary of the momentum polytope at the vertex labelled $Z$ and moved one of the two $A_{1}$-singularities in the total space to a non-toric position. The fact that the introduction of a singular point leads to an isomorphic family is a rather special phenomenon due to the large symmetry in this example.

3.3. One singular point II: Blow-up of $\mathbb{P}^{2}$. A similar tropical manifold, which we denote $B_{2}$, leads to a degeneration of the blow-up of $\mathbb{P}^{2}$ (Figure 3.3). Note that the boundary of $B_{2}$ is straight at both vertices labelled $U$ and $W$. Again we take $\varphi$
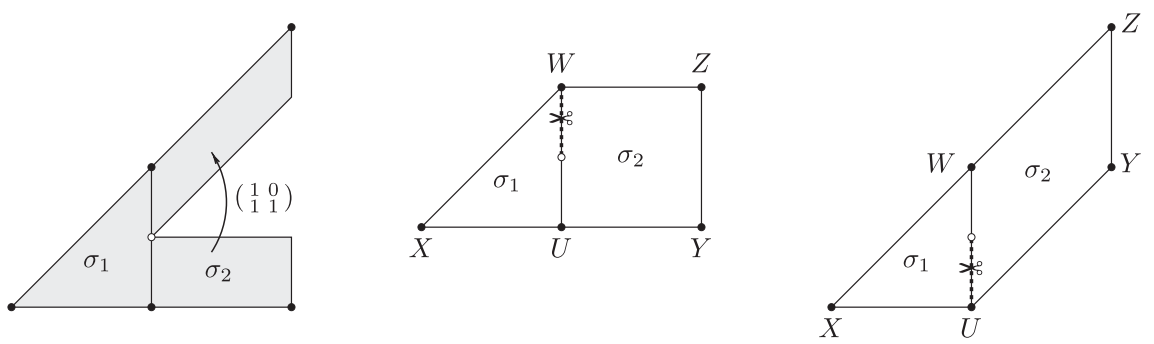

Figure 3.3. Three charts defining the tropical manifold $B_{2}$. 

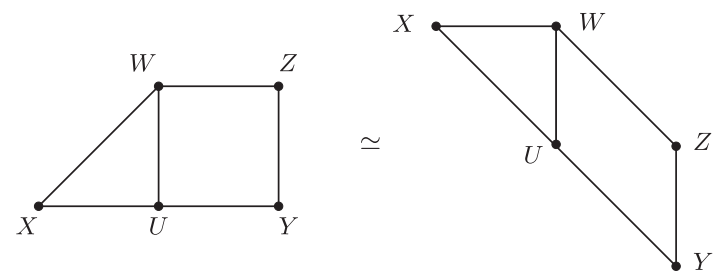

FiguRE 3.4. Torically constructed degeneration of $\mathbb{P}^{2}$ blown up once

to change slope by 1 along the only interior edge, with vertices labelled $U$ and $W$. There are 5 integral points, so we work in $\mathbb{P}_{\mathbb{A}^{1}}^{4}=\operatorname{Proj}(\mathbb{C}[t][X, Y, Z, U, W])$.

Now $\sigma_{2}$ is not a standard simplex. Thus we have a relation that does not receive any corrections from monodromy, namely

$$
W Y-U Z=0 .
$$

The gluing computations at the vertices labelled $U$ and $W$ homogenize to the two equations

$$
\begin{aligned}
X Y-t(\alpha U+W) U & =0, \\
X Z-t(\alpha U+W) W & =0 .
\end{aligned}
$$

For $U W \neq 0$ these are related by the substitution $Z=W Y / U$. The central fibre $X_{0}$ has two irreducible components $\mathbb{P}^{2}$ and $\mathbb{P}^{1} \times \mathbb{P}^{1}$, glued along a $\mathbb{P}^{1}$. The total space has only one singular point, the $A_{1}$-singularity at $[0,0,0,1,-\alpha] \in\left(X_{0}\right)_{\text {sing }}=\mathbb{P}^{1}$. The general fibre of this degeneration $\pi: X \rightarrow \mathbb{A}^{1}$ contains the (-1)-curve $E=V(X, \alpha U+W, \alpha Y+$ $Z$ ) whose contraction yields a $\mathbb{P}^{2}$. This is a well-known example: The 3 relations form the $2 \times 2$-minors of the matrix

$$
\left(\begin{array}{ccc}
U & W & X \\
Y & Z & t(\alpha U+W)
\end{array}\right)
$$

which for $t=1$ describe the cubic scroll, see e.g. [Ha], Example 7.24.

Again, this example has a toric analogue (Figure 3.4). The relations are

$$
W Y-U Z=0, \quad X Y-t U^{2}=0, \quad X Z-t U W=0 .
$$

In fact, the substitution $U \mapsto \alpha U+W, Y \mapsto \alpha Y+Z$ yields the non-toric ideal above.

3.4. Propagation. Once we start changing the gluing of components somewhere we are forced to change at other places as well to keep consistency. Thus in a sense the gluing functions propagate.

EXAMPLE 3.1. Consider the non-compact example of a tropical manifold with 4 maximal cells $\sigma_{1}, \ldots, \sigma_{4}$ shown in Figure 3.5. If we take the piecewise affine function $\varphi$ with $\varphi(1,0)=1, \varphi(0,1)=1, \varphi(-1,0)=0, \varphi(0,-1)=0$, the toric local model at $v$ is

$$
\mathbb{C}[t] \longrightarrow \mathbb{C}[x, y, z, w] /(x y-z w), \quad t \longmapsto x y=z w .
$$



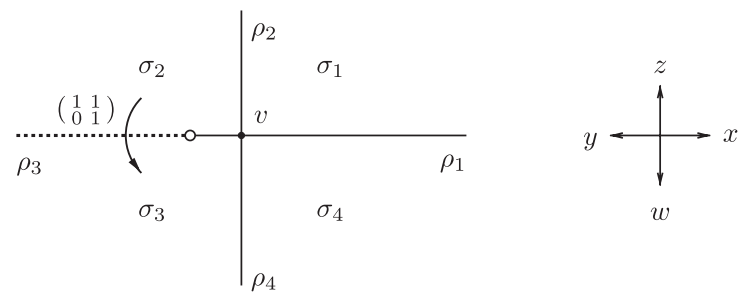

FiguRE 3.5. A tropical manifold forcing propagation of the gluing function. The figure on the right shows the tangent vectors belonging to the generators of the toric model.

In terms of integral tangent vectors at $v$ the generators are

$$
x=z^{(1,0,1)}, \quad y=z^{(-1,0,0)}, \quad z=z^{(0,1,1)}, \quad w=z^{(0,-1,0)} .
$$

Now let us try to glue together the $k$-th order neighbourhood of $X_{0}$ following the general procedure of $\S 2.4$ with gluing function $f_{\rho_{3}, v}=1+y$ and all other $f_{\rho_{i}, v}=1$. We have 4 rings $R_{v \rightarrow \sigma_{i}, \sigma_{i}}^{k}$ defining the thickenings of the irreducible components $X_{\sigma_{i}}, 4 \cdot 2=$ 8 rings for the one-dimensional toric strata $R_{v \rightarrow \rho_{i}, \sigma_{i}}^{k}, R_{v \rightarrow \rho_{i}, \sigma_{i-1}}^{k}(i$ taken modulo 4$)$, and 4 rings, identified mutually via an affine chart at $v$,

$$
R_{v \rightarrow v, \sigma_{i}}^{k}=\mathbb{C}[x, y, z, w, t] /\left((x y-z w, x y-t)+I_{k}\right)
$$

for the thickening of the zero-dimensional toric stratum $X_{v}$. Here $I_{k}=(x, z)^{k+1}+$ $(x, w)^{k+1}+(y, z)^{k+1}+(y, w)^{k+1}$ is the ideal generated by monomials that are divisible by $t^{k+1}$ at the generic point of $X_{\sigma_{i}}$ for some $i$. For the toric local model we want to take the inverse limit of all these rings with respect to the homomorphisms that we have introduced between them. These are of two kinds. First, for $\tau^{\prime} \subseteq \tau$ we have $R_{v \rightarrow \tau, \sigma_{i}}^{k} \rightarrow R_{v \rightarrow \tau^{\prime}, \sigma_{i}}^{k}$ defining the inclusion of toric strata with reference cell $\sigma_{i}$. Second, there are the change of reference cell isomorphisms $R_{v \rightarrow \tau, \sigma_{i}}^{k} \rightarrow R_{v \rightarrow \tau, \sigma_{i \pm 1}}^{k}$. This requires compatibility of the compositions. In the present case this comes down to checking the following. Let $\overline{\theta_{v}^{k}}$ be the composition

$$
R_{v \rightarrow v, \sigma_{1}}^{k} \longrightarrow R_{v \rightarrow v, \sigma_{2}}^{k} \longrightarrow R_{v \rightarrow v, \sigma_{3}}^{k} \longrightarrow R_{v \rightarrow v, \sigma_{4}}^{k} \longrightarrow R_{v \rightarrow v, \sigma_{1}}^{k}
$$

of changing the reference cell $\sigma_{i}$ counterclockwise around the origin by crossing the $\rho_{i}$. The compatibility condition is $\overline{\theta_{v}^{k}}=\mathrm{id}$. But crossing $\rho_{1}, \rho_{2}$ and $\rho_{4}$ yields the identity. Thus $\overline{\theta_{v}^{k}}$ equals the change of reference cell isomorphism from crossing $\rho_{3}$, which is

$$
x \mapsto x, \quad y \mapsto y, \quad z \mapsto(1+y) z, \quad w \mapsto(1+y)^{-1} w
$$

Recall that we pick up a negative power of $f_{\rho_{3}, v}=1+y$ if the tangent vector points from $\rho_{3}$ into $\sigma_{3}$, while monomials with exponents tangent to $\rho_{3}$ are left invariant. 
Note also that because $y$ is nilpotent now, $1+y$ is invertible, so this is a well-defined automorphism of $R_{v \rightarrow v, \sigma_{1}}^{k}$. In any case, we see $\overline{\theta_{v}^{k}} \neq \mathrm{id}$ as soon as $k \geq 2$.

To fix this it is clear how to proceed: Just take $f_{\rho_{1}, v}=1+y$ as well. Then by the sign conventions, crossing $\rho_{1}$ gives the inverse of what we had for $\rho_{3}$. Then $\overline{\theta_{v}^{k}}=\mathrm{id}$ as automorphism of $R_{v \rightarrow v, \sigma_{i}}^{k}$ for any $k$ (and any $i$ ).

REMARK 3.2. There is an important observation to be made here. On $X_{\rho_{3}}$ the monomial $y$ is nonzero, that is, of order zero with respect to $t$. But once we move to a general point of $X_{\rho_{1}}$ we have $y=x^{-1} t$ because of the relation $x y=t$. Thus the $t$-order increases. And this is not an accident, but happens whenever we follow a monomial $z^{(m, h)},(m, h) \in \Lambda_{v} \times \mathbb{Z}$ in direction $-m([\mathbf{G r S i 2}]$, Proposition 2.6). More precisely, if $\varphi$ differs by $a_{\rho} \in \Lambda_{\rho}^{\perp}$ along a codimension one cell $\rho$ then a monomial with tangent vector $m$ passing through $\rho$ changes $t$-order by $-\left\langle m, a_{\rho}\right\rangle$.

This implies that even in examples where the invariant line $\ell$ emanating from a singular point is unbounded, the gluing functions always converge $t$-adically. Said differently, for the construction of a $k$-th order deformation of $X_{0}$, we need to propagate the contribution of each singular point only through finitely many cells.

Of course, there is no reason for the propagation to stay in the 1-skeleton of $\mathscr{P}$. The gluing functions then separate from the $(n-1)$-skeleton of $\mathscr{P}$ and move into the interiors of maximal cells.

ExAmple 3.3. Let us modify Example 3.1 as shown in Figure 3.6 with $\varphi(-1,0)=$ $0, \varphi(0,-1)=0, \varphi(1,1)=1$. The gluing functions are $f_{\rho_{2}, v}=1+x$ and all other $f_{\rho_{i}, v}=$ 1. The toric local model at $v$ is now the normal crossing degeneration

$$
\mathbb{C}[t] \longrightarrow \mathbb{C}[x, y, z], \quad t \longmapsto x y z
$$

with $x=z^{(-1,0,0)}, y=z^{(0,-1,0)}, z=z^{(1,1,1)}$. Following the gluing isomorphisms around $v$ leads to the automorphism

$$
\overline{\theta_{v}^{k}}: x \mapsto x, \quad y \mapsto(1+x)^{-1} y, \quad z \mapsto(1+x) z
$$

of the ring

$$
R_{v \rightarrow v, \sigma_{i}}^{k}=\mathbb{C}[x, y, z, t] /\left(x y z-t, x^{k+1}, y^{k+1}, z^{k+1}\right) .
$$

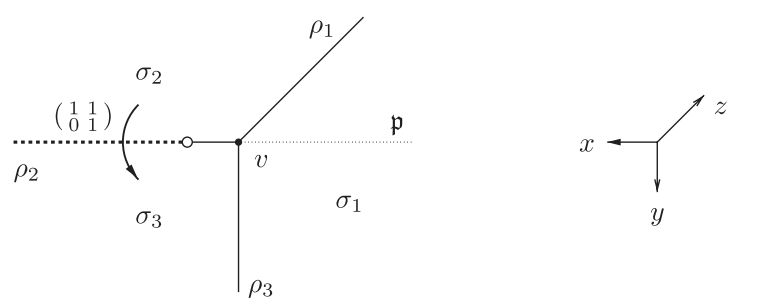

Figure 3.6. Propagation of the gluing function into a maximal cell. The figure on the right shows the tangent vectors belonging to the generators of the toric model. 
For $k>0$ this is again not the identity, and we can't fix this by changing any of the gluing functions. But since $1+x$ is invertible on the whole thickening $\operatorname{Spec}(\mathbb{C}[x, y, z, t] /$ $\left.\left(x y z-t, x^{k+1}\right)\right)$ of $X_{\sigma_{1}}$, the inverse of $\overline{\theta_{v}^{k}}$ can be viewed as an automorphism of $X_{\sigma_{1}}$ that propagates along the ray $\mathfrak{p}=\mathbb{R}_{\geq 0} \cdot(1,0)$ emanating from the codimension two cell $v$. We then change the gluing procedure by taking one copy of the thickening of $X_{\sigma_{1}}$ for each connected component of $\sigma_{1} \backslash \mathfrak{p}$. Passing through $\mathfrak{p}$ means applying the attached automorphism. The thickenings of the codimension one strata $X_{\rho_{1}}$ and $X_{\rho_{3}}$ are viewed as closed subschemes of the copies labelled by the connected component of $\sigma_{1} \backslash \mathfrak{p}$ containing $\rho_{1}$ and $\rho_{3}$, respectively.

Inserting $\mathfrak{p}$ makes the gluing consistent to all orders. The result is isomorphic to $\operatorname{Spec}(\mathbb{C}[x, y, z, t] /(x y z-(1+x) t))$.

Apart from the gluing functions we have now introduced another object, automorphisms propagating along rays. The higher-dimensional generalization of rays are walls ([GrSi2], Definition 2.20): They are one-codimensional polyhedral subsets of some maximal cell $\sigma$, emanating from a two-codimensional polyhedral subset $\mathfrak{q}$ into the interior of $\sigma$, and extending all the way through $\sigma$ along some integral tangent vector $-m_{\mathfrak{p}}$, that is, $\mathfrak{p}=\sigma \cap\left(\mathfrak{q}-\mathbb{R}_{\geq 0} \cdot m_{\mathfrak{p}}\right)$. The attached automorphism is of the form

$$
z^{(m, h)} \longmapsto\left(1+c_{\mathfrak{p}} z^{\left(m_{\mathfrak{p}}, h_{\mathfrak{p}}\right)}\right)^{\left\langle m, n_{\mathfrak{p}}\right\rangle} \cdot z^{(m, h)},
$$

where $h_{\mathfrak{p}}>0$ is the $t$-order of the attached monomial and $c_{\mathfrak{p}} \in \mathbb{C}$. We will discuss how to obtain these walls systematically in the context of scattering in Section 4.

3.5. Several singular points: The non-interacting case. For several singular points $P_{1}, \ldots, P_{l} \in B$ the monodromy invariant direction determines an affine line $\ell_{\mu}$ emanating from each $P_{\mu}$. As long as these coincide or do not intersect, the construction presented so far works.

EXAMPLE 3.4. Here is an example with two singular points having parallel invariant lines $\ell_{1}, \ell_{2}$, see Figure 3.7. We will see that, depending on a choice of parameter, it leads to a degeneration either of the Hirzebruch surface $\mathbb{F}_{2}=\mathbb{P}\left(\mathcal{O}_{\mathbb{P}^{1}}(-2) \oplus \mathcal{O}_{\mathbb{P}^{1}}\right)$ or of $\mathbb{P}^{1} \times \mathbb{P}^{1}$.

In this case we have two codimension one strata along which we glue. Taking $\varphi$ to change slope by 1 along each interior edge again, the homogenization of the gluing along codimension one cells gives the polynomials

$$
X V-t(U+R) U, X S-t(R+U) R, U Y-t(V+\lambda S) V, R Y-t(\lambda S+V) S .
$$

Here $\lambda \in \mathbb{C}^{*}$ is a parameter that we can not get rid of by automorphism. Note that $\lambda$ determines the relative position of the zero loci of the gluing functions when compared in $X_{\sigma_{2}}=\mathbb{P}^{1} \times \mathbb{P}^{1}$. There is one more toric relation $R V-S U=0$ from $\sigma_{2}$. The corresponding subscheme of $\mathbb{P}_{\mathbb{A}^{1}}^{5}=\operatorname{Proj}_{\mathbb{C}[t]}(\mathbb{C}[t][X, Y, R, S, U, V])$ has one extra component $V(R, S, U, V)$. In fact, saturating with respect to any of $R, S, U, V$ gives another relation $X Y-t^{2}(U+R)(V+\lambda S)=0$. This relation can also be deduced directly from following the line connecting the vertices $X$ and $Y$ in the chart shown in Figure 3.7 on the right. 

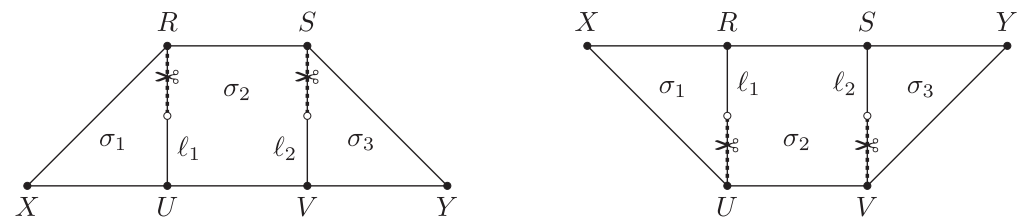

FiguRE 3.7. Two charts for a tropical manifold with two parallel invariant lines $\ell_{1}, \ell_{2}$.
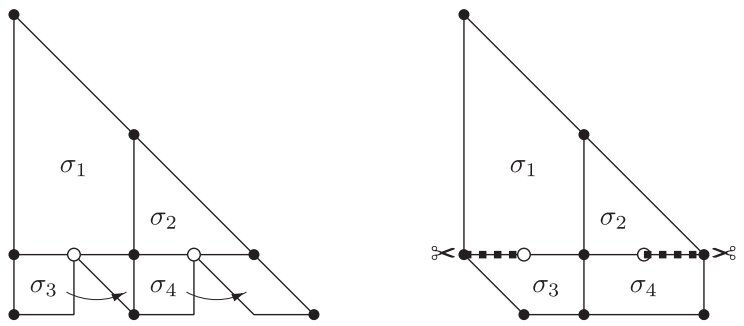

FiguRE 3.8. A tropical manifold for a degeneration of $\mathbb{P}^{2}$ blown up twice. The invariant lines emanating from the two singular points agree.

These six polynomials are the $2 \times 2$ minors of the matrix

$$
\left(\begin{array}{cccc}
X & t(V+\lambda S) & U & R \\
t(U+R) & Y & V & S
\end{array}\right)
$$

For fixed $t \neq 0$, this is a scroll in $\mathbb{P}^{5}$ whose ruling is given by the lines whose equations are linear combinations of the two rows of the above matrix. The image of this scroll under the rational map $\kappa: X_{t} \rightarrow \mathbb{P}^{3}$ given by $[x, y, u, v, r, s] \mapsto\left[x_{0}, x_{1}, x_{2}, x_{3}\right]=[u+$ $r, y, v, s]$ has equation $X_{0} X_{1}-t\left(X_{2}+X_{3}\right)\left(X_{2}+\lambda X_{3}\right)=0$ in $\mathbb{P}^{3}$. For $\lambda \neq 1$ this is a smooth quadric and $\kappa$ induces an isomorphism $X_{t} \simeq \mathbb{P}^{1} \times \mathbb{P}^{1}$. For $\lambda=1$ the quadric $X_{0} X_{1}-t\left(X_{2}+X_{3}\right)^{2}=0$ has an $A_{1}$-singularity, $\kappa$ contracts the $(-2)$-curve given by $Y=V+S=U+R=X=0$, and $X_{t} \simeq \mathbb{F}_{2}$.

EXAMPLE 3.5. Figure 3.8 features two singularities with the same invariant line. The general fibre of the corresponding degeneration is a $\mathbb{P}^{2}$ blown up in two different points. We don't bother to write down the homogeneous equations.

This example is easy to generalize to any number of blown up points, which then gives our first genuinely non-toric examples.

Many more examples can be obtained from $[\mathbf{S y}]$, which contains a toolkit for the construction of two-dimensional affine manifolds with focus-focus singularities. The symplectic 4-manifold constructed in this reference from such a singular affine manifold is a symplectic model for the general fibre of our degeneration. 

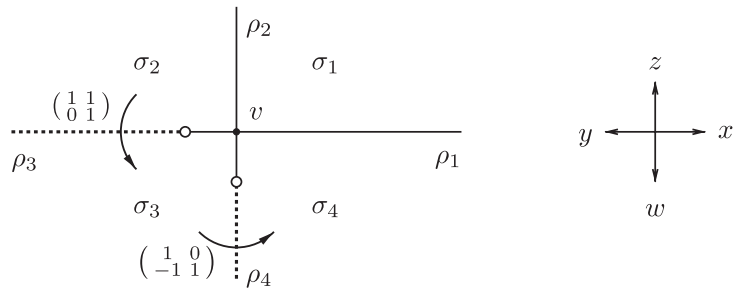

Figure 4.1. A tropical manifold leading to inconsistent gluing. The figure on the right shows the tangent vectors belonging to the generators of the toric model.

\section{Scattering}

If the invariant lines emanating from singular points of a two-dimensional affine structure intersect, the gluing construction becomes inconsistent again.

4.1. First example of scattering. We study the modification of Example 3.1 shown in Figure 4.1, with gluing functions

$$
f_{\rho_{1}, v}=1+y=1+x^{-1} t, \quad f_{\rho_{2}, v}=1+w=1+z^{-1} t, \quad f_{\rho_{3}, v}=1+y, \quad f_{\rho_{4}, v}=1+w .
$$

Following the gluing isomorphisms of $R_{v \rightarrow v, \sigma_{i}}^{k}$ by going counterclockwise around $v$, with starting cell $\sigma_{1}$ say, yields

$$
\begin{aligned}
x & \longmapsto(1+w) x \\
& \longmapsto\left(1+(1+y)^{-1} w\right) x \\
& \longmapsto\left(1+(1+(1+w) y)^{-1} w\right)(1+w)^{-1} x \\
& \longmapsto\left(1+(1+(1+(1+y) w) y)^{-1}(1+y) w\right)(1+(1+y) w)^{-1} x \\
& =\left(1+((1+y)+(1+y) w y)^{-1}(1+y) w\right)(1+(1+y) w)^{-1} x \\
& =\left(1+(1+w y)^{-1} w\right)(1+w+w y)^{-1} x \\
& =((1+w y)+w)(1+w y)^{-1}(1+w+w y)^{-1} x=(1+w y)^{-1} x,
\end{aligned}
$$

and similarly,

$$
y \longmapsto(1+w y) y, \quad z \longmapsto(1+w y) z, \quad w \longmapsto(1+w y)^{-1} w .
$$

Thus we can again achieve $\overline{\theta_{v}^{k}}=$ id for all $k$ by inserting the ray $\mathfrak{p}=\mathbb{R}_{\geq 0} \cdot(1,1)$ with attached function $1+w y=1+t^{2} x^{-1} z^{-1}$ !

4.2. A projective example. Of course, this example can also be made projective, say as in Figure 4.2. The ideal defining the degeneration is generated by the two polynomials

$$
X Y-t(U+W) U, \quad Z W-t(U+Y) U
$$




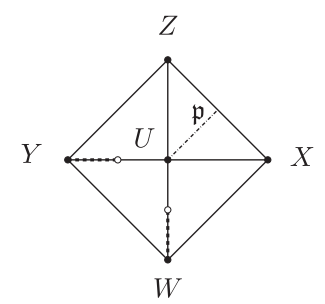

Figure 4.2. Chart for the tropical manifold $B_{3}$. The dashed lines denote cuts.

These relations can be most simply derived from gluing $k$-th order standard thickenings of $X_{\sigma_{3}}, X_{\sigma_{4}}$ and of $X_{\sigma_{2}}, X_{\sigma_{3}}$, respectively, and let $k$ tend to infinity. This procedure does not seem to depend on $\mathfrak{p}$. In fact, in this example, the insertion of $\mathfrak{p}$ merely assures that the same relations are obtained with any other pairs of neighbouring maximal cells.

As an abstract deformation this is isomorphic to the toric one with ideal ( $X Y-$ $\left.t U^{2}, Z W-t Y^{2}\right)$ presented in Example 1.4.

4.3. Systematic procedure for the insertion of walls. The long computation for the insertion of the ray $\mathfrak{p}$ in Example 4.1 looks like quite an accident. But there is a systematic procedure to insert rays (or walls in higher dimension) to achieve consistency to any finite order. The number of rays to be inserted becomes arbitrarily large with increasing $k$, however, essentially with the only exception the example discussed in $\S 4.1$. This procedure originates from the two-dimensional situation of $[\mathbf{K o S o}]$. Of course, due to the non-discrete nature of the discriminant locus the higher dimensional situation is much more involved.

Systematically we proceed as follows. By induction one arrives at a collection of walls and modifications of the gluing functions, a structure (in its architectural meaning) $\mathscr{S}$ as we call it. A structure consists of walls and so-called slabs. A slab $\mathfrak{b}$ is a polyhedral subset of some $\rho \in \mathscr{P}^{[n-1]}$ together with a collection $f_{\mathfrak{b}, v}$ of higher order corrections of the gluing functions $f_{\rho, v}$, one for each vertex $v \in \rho$ that is contained in a connected component of $\rho \backslash \Delta$ intersecting $\mathfrak{b}$. These fulfill the same change of vertex formula (2.3) as the $f_{\rho, v}$, but interpreted in the rings $R_{\rho \rightarrow \rho, \sigma}^{k}$ for any $\sigma \in \mathscr{P}_{\max }$ containing $\rho$. We need slabs because a wall hitting a codimension one cell $\rho$ may lead to different corrections on both sides of the wall. Technically we refine $\mathscr{P}$ to a polyhedral decomposition $\mathscr{P}_{\mathscr{S}}$ in such a way that the walls lie in the $(n-1)$-skeleton of $\mathscr{P}_{\mathscr{S}}$. Then the underlying subsets of the slabs are exactly the $(n-1)$-cells of $\mathscr{P}_{\mathscr{S}}$ contained in the $(n-1)$-skeleton of $\mathscr{P}$.

For consistency of the gluing construction it suffices to follow loops around the codimension two cells of $\mathscr{P}_{\mathscr{S}}$, the joints of the structure (or rather of $\mathscr{P}_{\mathscr{S}}$ ). This is shown in [GrSi2], Lemma 2.30; the precise definition of consistency is in Definition 2.28. The fact that the gluing construction for a structure consistent to order $k$ gives a $k$-th order toric degeneration occupies $\S 2.6$ in $[\mathbf{G r S i 2}]$.

Thus consistency is really a codimension two feature. For the scattering computation at a joint $\mathfrak{j}$ we can therefore work over the ring $S=\mathbb{C} \llbracket t \rrbracket\left[\Lambda_{\mathfrak{j}}\right]$ of Laurent 
polynomials with coefficients in $\mathbb{C} \llbracket t \rrbracket$ and exponents in $\Lambda_{\mathfrak{j}}$, the lattice of integral tangent vectors along $\mathfrak{j}$. In fact, if $\overline{\theta_{\mathfrak{j}}}$ is the composition of the gluing morphisms following a small loop around such a joint, then $\overline{\theta_{\mathrm{j}}}$ is the identity on any monomial $z^{(m, h)}$ with $m$ tangent to $\mathfrak{j}$.

By making the discriminant locus $\Delta$ sufficiently non-rational or by deforming it a little, we can assume the intersection of $\Delta$ with $\mathfrak{j}$ to be of codimension one in $\mathfrak{j}$. Let $p \in \mathfrak{j} \backslash \Delta$ be a general point and denote by $\sigma_{\mathfrak{j}} \in \mathscr{P}$ the smallest cell containing $\mathfrak{j}$. Note that $\sigma_{\mathfrak{j}}$ has dimension $n-2, n-1$ or $n$. The computations take place in an affine chart at a vertex $v \in \sigma_{\mathfrak{j}}$ in the same connected component of $\sigma_{\mathfrak{j}} \backslash \Delta$ as $p$. In an affine chart at $v$ decompose the lattice of integral tangent vectors by choosing a complement $\bar{\Lambda} \simeq \mathbb{Z}^{2}$ to $\Lambda_{\mathfrak{j}} \subseteq \Lambda_{v}$

$$
\Lambda_{v}=\Lambda_{\mathfrak{j}} \oplus \bar{\Lambda}
$$

While the procedure essentially is always the same, the setup for the scattering differs somewhat depending on $\operatorname{dim} \sigma_{\mathfrak{j}} \in\{n-2, n-1, n\}$. For being explicit let us assume $\operatorname{dim} \sigma_{\mathfrak{j}}=n-2$, which is is the most difficult case; it is also the situation for the initial scattering of codimension one cells of $\mathscr{P}$ intersecting in a codimension two cell $\mathfrak{j}=\sigma_{\mathfrak{j}}$ of $\mathscr{P}$. At a later stage $(k>0)$ the inclusion $\mathfrak{j} \subseteq \sigma_{\mathfrak{j}}$ may be strict and there may be additional walls running into $\mathfrak{j}$ from scatterings at other joints.

The scattering procedure is a computation in the rings $R_{\sigma_{j} \rightarrow \sigma_{j}, \sigma}^{k}$ using induction on $k$. However, these rings are not practical for computations with a computer algebra system, and they would require the introduction of log structures in Example 4.1. Log structures are necessary to make things work globally in the end, but on the level of this survey it does not seem appropriate to get into these kinds of technicalities. We therefore present the scattering computation in a traditional polynomial ring.

For any order $k^{\prime} \geq 0$ the composition of gluing morphisms around $\mathfrak{j}$, starting at a reference cell $\sigma \in \mathscr{P}_{\max }$, defines an automorphism $\overline{\theta_{\mathfrak{j}}^{k^{\prime}}}$ of the ring $R_{\sigma_{j} \rightarrow \sigma_{j}, \sigma}^{k^{\prime}}$. This ring is a finite $S_{f}$-algebra, where $f=\prod_{\rho \supseteq \sigma_{j}} f_{\rho, v}$. Let $\bar{\varphi}$ be the restriction to $\{0\} \times \bar{\Lambda} \simeq \mathbb{Z}^{2}$ of a non-negative representative of $\varphi$. By changing the representative by an affine function we can assume

$$
\bar{\varphi}(0,0)=\bar{\varphi}(-1,0)=0, \quad 0 \leq \bar{\varphi}(0,1) \leq \bar{\varphi}(1,1)
$$

which by convexity and integrality of $\bar{\varphi}$ implies

$$
\bar{\varphi}(1,1) \geq 1 \text {. }
$$

Letting $x:=z^{(-1,0,0)}, y:=z^{(0,-1,0)}, z:=z^{(1,1,1)}$ this exhibits $R_{\sigma_{j} \rightarrow \sigma_{j}, \sigma}^{k^{\prime}}$ as a quotient of an $S_{f}$-subalgebra of

$$
R^{k}:=S_{f}[x, y, z] /\left((x y z-t)+(x, y, z)^{k+1}\right),
$$

for some $k \geq k^{\prime}$. The smallest possible $k$ is the minimum of the degrees $a+b+c$ for $x^{a} y^{b} z^{c}$ having $\sigma_{\mathfrak{j}}$-order (introduced in $\S 3.1$ ) larger than $k^{\prime}$. Conversely, any computation in $R^{k}$ can be obtained from a computation in $R_{\sigma_{j} \rightarrow \sigma_{j}, \sigma}^{k^{\prime}}$ for $k^{\prime} \gg 0$. Thus the result of the scattering procedure is the same with both sorts of rings. 
Initially the only scattering happens at codimension two cells $\tau \in \mathscr{P}$. The starting data are the gluing functions $f_{\rho, v}$ for all codimension one cells $\rho \supseteq \tau$ where $v \in \tau$ is some vertex selecting an affine chart for the computation. Consistency for $k=1$ already requires a compatibility condition for the restrictions $\left.f_{\rho, v}\right|_{X_{\tau}}$ that we can see by studying the following local example.

EXAMPLE 4.1. To consider a local scattering situation for $k=1$ take $\mathfrak{j}=\tau=$ $\mathbb{R}^{n-2} \times\{0\} \subseteq \mathbb{R}^{n}$ as an affine subspace and let $\rho_{i}=\tau \times \mathbb{R}_{\geq 0} m_{i}, i=1, \ldots, l$, be affine half-hyperplanes emanating from $\tau$ in directions $m_{i} \in \mathbb{Z}^{2}=\bar{\Lambda}$ in the normal lattice $\mathbb{Z}^{n} / \Lambda_{\tau}=\bar{\Lambda}$. (Strictly speaking this is not a legal situation because $\tau$ does not have a vertex, but this is irrelevant for the present discussion.) We assume the $\rho_{i}$ labelled cyclically around $\tau$, that is, $\rho_{i}$ separates two maximal cell $\sigma_{i-1}, \sigma_{i}$ containing $\tau$, for any $i \in \mathbb{Z} / l \mathbb{Z}$. Let $n_{i}=\left(a_{i}, b_{i}\right)$ be a primitive normal vector to $\Lambda_{\rho_{i}}$ that evaluates non-negatively on $\sigma_{i}$. Then passing from $\sigma_{i-1}$ to $\sigma_{i}$ gives the automorphism

$$
\overline{\theta_{i}^{1}}: z^{m} \longmapsto f_{\rho_{i}}^{-\left\langle n_{i}, m\right\rangle} \cdot z^{m}
$$

of the ring $R^{1}=S_{f}[x, y, z] /(x, y, z)^{2}$ from (4.1). Because in this local example there is no vertex we drop the reference vertex from the notation for the gluing functions $f_{\rho_{i}}$. The loop around the joint $\tau$ gives the automorphism $\overline{\theta_{\tau}^{1}}=\overline{\theta_{k}^{1}} \circ \ldots \overline{\theta_{1}^{1}}$. Because we work modulo $(x, y, z)^{2}$ the effect of applying $\overline{\theta_{k}^{i}}$ to a monomial is multiplication with a power of the restriction $\left.f_{\rho_{i}}\right|_{X_{\tau}} \in S_{f}$. We obtain

$$
\overline{\theta_{\tau}^{1}}(x)=\left(\prod_{i} f_{\rho_{i}}^{-\left\langle\left(a_{i}, b_{i}\right),(-1,0)\right\rangle}\right) \cdot x=\left(\prod_{i}\left(f_{\rho_{i}} \mid X_{\tau}\right)^{a_{i}}\right) \cdot x,
$$

and analogously,

$$
\overline{\theta_{\tau}^{1}}(y)=\left(\prod_{i}\left(\left.f_{\rho_{i}}\right|_{X_{\tau}}\right)^{b_{i}}\right) \cdot y, \quad \overline{\theta_{\tau}^{1}}(z)=\left(\prod_{i}\left(f_{\rho_{i}} \mid X_{\tau}\right)^{-a_{i}-b_{i}}\right) \cdot z .
$$

Thus consistency for $k=1$ requires the following multiplicative condition for the slab functions

$$
\prod_{i}\left(f_{\rho_{i}} \mid X_{\tau}\right)^{b_{i}}=1 \quad \text { and } \quad \prod_{i}\left(f_{\rho_{i}} \mid X_{\tau}\right)^{a_{i}}=1 .
$$

Example 4.1 shows that apart from the change of vertex formula (2.3), slab functions need to fulfill a number of algebraic equations to achieve consistency for $k=1$. More precisely, each $\tau \in \mathscr{P}^{[n-2]}$ yields two multiplicative equations as in (4.2) for the restrictions $\left.f_{\rho, v}\right|_{X_{\tau}}$ of the slab functions for those $\rho \in \mathscr{P}[n-1]$ that contain $\tau$, see Equation (1.10) in [GrSi2] for the general form. Note that by the change of vertex formula the relations obtained from different vertices $v, v^{\prime} \in \tau$ are equivalent.

For those readers familiar with [GrSi1], what this shows is that specifying the gluing functions $f_{\rho, v}$ for $k=1$ is essentially the same as specifying a log structure on $X_{0}$ (cf. [GrSi1], Theorem 3.22). Thus the log structures of [GrSi1] on $X_{0}$ can be viewed as "initial conditions" for our construction.

To explain the next steps $(k>1)$ let us look at the following typical scattering situation in dimension 3 . Let the reference vertex be $v=0 \in \mathbb{R}^{3}$ and let the codimension 
two cell $\tau$ be contained in the coordinate axis $\mathbb{R} \cdot(0,0,1)$. We have three codimension one cells $\rho_{1}, \rho_{2}, \rho_{3} \supseteq \tau$ with $K_{\tau} \rho_{i} / T_{\tau}$ spanned by $(-1,0,0),(-1,-3,0)$ and $(2,3,0)$, respectively. For each $i$ there is a unique slab $\mathfrak{b}_{i} \subseteq \rho_{i}$ containing $x$. The piecewise affine function $\varphi$ is uniquely defined by $\left.\varphi\right|_{\tau}=0$ and

$$
\varphi(-1,0,0)=\varphi(-1,-3,0)=0, \quad \varphi(2,3,0)=3 .
$$

Following the maximal cells $\sigma_{1}, \sigma_{2}, \sigma_{3}$ containing $\tau$ counterclockwise, with $\sigma_{1}$ containing $\rho_{1}$ and $\rho_{2}$, we have $\varphi(a, b)=0,3 a-b$ and $b$ for $(a, b) \in \sigma_{1}, \sigma_{2}, \sigma_{3}$, respectively. In particular, $\varphi(2,3,0)=3$ is the smallest possible value making $\varphi$ integral and with $\left.\varphi\right|_{\sigma_{1}}=0$. The exponents of our monomials take values in

$$
P:=\{(m, h) \in \Lambda \times \mathbb{Z} \mid h \geq \varphi(m)\} .
$$

As slab functions take

$$
\begin{aligned}
f_{\mathfrak{b}_{1}, v} & =1+z^{(0,0,1,0)}+2 z^{(-1,0,0,1)} \\
f_{\mathfrak{b}_{2}, v} & =1+z^{(0,0,1,0)}-z^{(-1,-3,1,0)} \\
f_{\mathfrak{b}_{3}, v} & =1+z^{(0,0,1,0)}+5 z^{(2,3,0,3)}
\end{aligned}
$$

where the exponents lie in $\Lambda \times \mathbb{Z}, \Lambda=\Lambda_{v}$. Thus in $z^{(a, b, c, h)}$ the first two coordinates $(a, b)$ determine the direction in $\Lambda / \Lambda_{\tau}$, the third coordinate $c$ is for the toric parameter on $X_{\tau}$ and $h-\varphi(a, b)$ determines the divisibility by $t$.

We also assume there is one wall $\mathfrak{p}$ containing $\tau$ coming from some earlier step, say with $K_{\tau} \mathfrak{p} / T_{\tau}$ spanned by $(-1,1,0)$ and with associated function

$$
f_{\mathfrak{p}}=1+7 z^{(-1,1,0,2)}
$$

Our scattering problem can be visualized in the two-dimensional normal space to $\tau$ as in Figure 4.3 on the left.

For the explicit scattering computation we now go over to the $S_{f}$-algebra $R^{k}$ from (4.1). Here $S_{f}=\mathbb{C} \llbracket t \rrbracket\left[w, w^{-1}\right]_{1+w}$ with $w=z^{(0,0,1,0)}$, and

$$
x=z^{(-1,0,0,0)}, \quad y=z^{(0,-1,0,0)}, \quad z=z^{(1,1,0,1)} .
$$

Note that $x y z=t$ defines the $t$-algebra structure, and a monomial $x^{a} y^{b} z^{c} w^{d}$ represents an element of $P$ if and only if

$$
\varphi(c-a, c-b, d)=\max \{0, c-b, 3(c-a)-(c-b)\} \geq c .
$$

With these notations our input data is

$$
f_{\mathfrak{b}_{1}, v}=1+w+2 t x, \quad f_{\mathfrak{b}_{2}, v}=1+w-w x y^{3}, \quad f_{\mathfrak{b}_{3}, v}=1+w+5 x z^{3}, \quad f_{\mathfrak{p}}=1+7 t x^{2} z .
$$



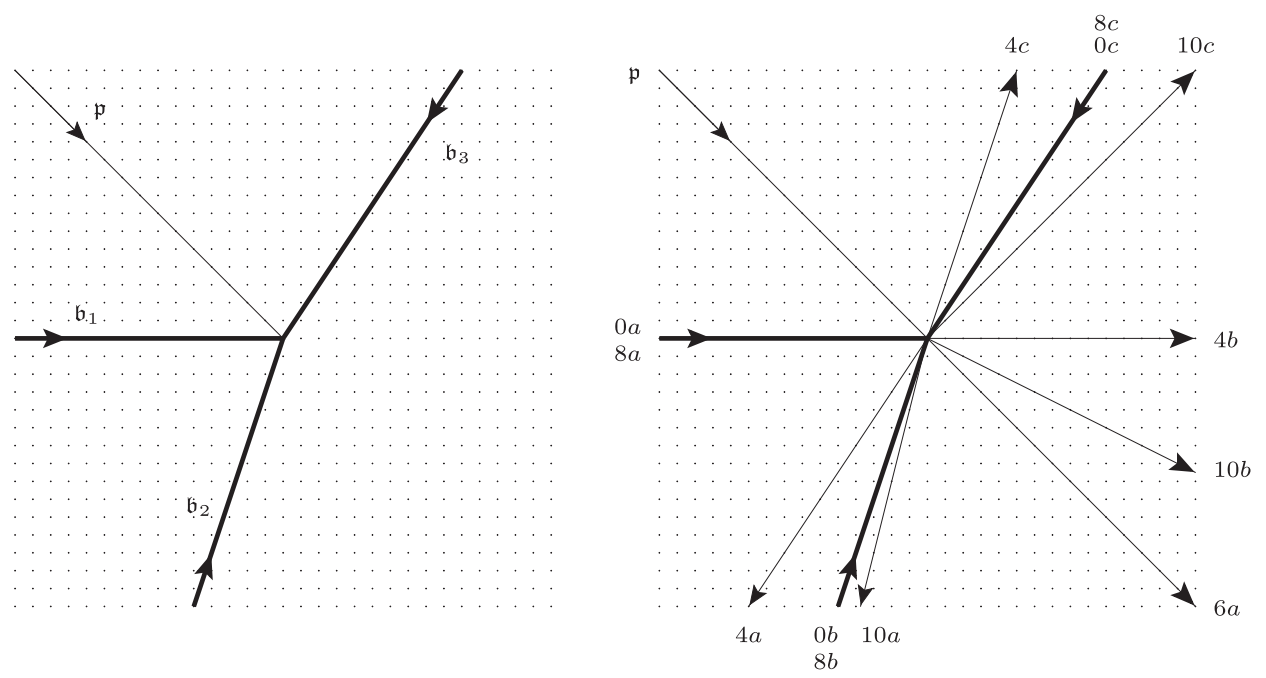

FiguRE 4.3. A scattering procedure: Starting data (left) and result at order 10. The labels are explained in the text.

It is now easy to compute the composition $\overline{\theta_{v}^{k}}$ by a computer algebra system. Seeing the various terms come up at higher order and getting rid of them by insertion of walls is very instructive. We therefore encourage the reader to do that and verify the following computations. Note that as we have seen in $\S 4.1$, the naive insertion procedure leads to huge expressions quickly, so a computation in $\mathbb{Q}[x, y, z, w]$ would be too slow. It is therefore important to expand in a Taylor series to order $k$ in each of $x, y$ and $z$ after each application of an automorphism associated to a slab or wall. Note also that to compute $\overline{\theta_{v}^{k}}$ as an automorphism of $R_{\tau \rightarrow \tau, \sigma_{1}}^{k}$ we have to observe the difference between the $\tau$-order of a monomial $x^{a} y^{b} z^{c}$ and its degree $a+b+c$. For example, both $x^{2} y$ and $x^{5} y^{5} z^{5}=t^{5}$ have $\tau$-order 5 , but $x^{2} y$ is already visible modulo $(x, y, z)^{4}$ while $x^{5} y^{5} z^{5}$ requires working modulo $(x, y, z)^{16}$.

The computation in $R^{k}$ gives consistency up to $k=3$. At degree 4 one has to insert three walls, denoted $4 a, 4 b, 4 c$ in Figure 4.3 on the right. The associated functions are

$$
1+5(1+w)^{2} x z^{3}, \quad 1+2(1+w)^{2} x^{2} y z, \quad 1-(1+w)^{2} w x y^{3} .
$$

This is just the part $f_{\mathfrak{b}_{i}}-(1+w)$ of the slab functions that do not cancel, with an appropriate power of $1+w$ that we will explain shortly. Similarly, at degree 6 the incoming wall $\mathfrak{p}$ starts contributing, forcing its continuation by a wall in direction $(1,-1)$ with function $1+7(1+w)^{5} t x^{2} z(6 a$ in Figure 4.3 on the right). Then everything goes well up to degree 8 , where we find interactions of two of such terms, namely some multiples of $x^{3} y z^{4}=t x^{2} z^{3}, x^{3} y^{4} z=t x^{2} y^{3}, x^{2} y^{3} z^{3}=t^{2} y z$. These monomials have directions $(1,3),(-2,-3),(1,0)$, so have to scatter away in the directions $(-1,-3),(2,3),(-1,0)$ occupied by the slabs. Since we do not want to allow walls to lie above slabs the only possibility is to change the slab functions. Here is the change 
that makes things consistent to degree 9:

$$
\begin{aligned}
& f_{\mathfrak{b}_{1}, v}^{\prime}=1+w+2 t x-15(1+w)^{2} t^{2} w y z, \\
& f_{\mathfrak{b}_{2}, v}^{\prime}=1+w-w x y^{3}+30(1+w)^{2} t x^{2} z^{3}, \\
& f_{\mathfrak{b}_{3}, v}=1+w+5 x z^{3}-6(1+w)^{2} w t x^{2} y^{3} .
\end{aligned}
$$

Let us go through degree 10 in detail. The composition modulo $(x, y, z)^{11}$ yields

$$
\begin{aligned}
& x \longmapsto\left(1-\frac{700 x^{4} y z^{5}}{1+w}-\frac{14 x^{5} y^{2} z^{3}}{1+w}-56(1+w)^{2} w x^{4} y^{4} z^{2}\right) \cdot x \\
& y \longmapsto\left(1+\frac{175 x^{4} y z^{5}}{1+w}-\frac{28 x^{5} y^{2} z^{3}}{1+w}+56(1+w)^{2} w x^{4} y^{4} z^{2}\right) \cdot y \\
& z \longmapsto\left(1+\frac{525 x^{4} y z^{5}}{1+w}+\frac{42 x^{5} y^{2} z^{3}}{1+w}\right) \cdot z .
\end{aligned}
$$

The occurring monomials are $x^{4} y z^{5}=t x^{3} z^{4}, x^{5} y^{2} z^{3}=t^{2} x^{3} z, x^{4} y^{4} z^{2}=t^{2} x^{2} y^{2}$, which have directions $(1,4),(-2,1)$ and $(-1,-1)$, respectively. (The fact that the last one points in the same direction as $z$ explains why it does not show up in the last line: It belongs to a wall acting trivially on $z$.) Thus we want to insert walls in directions $(-1,-4),(2,-1)$ and $(1,1)(10 a, 10 b$ and $10 c$ in Figure 4.3) with functions

$$
1+a t x^{3} z^{4}, \quad 1+b t^{2} x^{3} z, \quad 1+c t^{2} x^{2} y^{2},
$$

for some $a, b, c \in \mathbb{C}[w]$. We now explain how to determine the coefficients $a, b, c$. Since they come in a product with monomials of degree 10, they do not interact with any monomial of non-zero degree. Hence the influence to the composition can be computed from a scattering diagram with only the wall in question and the three slabs $\mathfrak{b}_{i}$ with functions $f_{\mathfrak{b}_{i}}=1+w, i=1,2,3$. Here is the computation of the composition for the wall 10a:

$$
\begin{aligned}
x & \stackrel{\mathfrak{b}_{1}}{\longmapsto} x \stackrel{\mathfrak{b}_{2}}{\longmapsto}(1+w)^{3} x \stackrel{10 a}{\longmapsto}\left(1+a t x^{3} z^{4}\right)^{4} \cdot(1+w)^{3} x \\
& \stackrel{\mathfrak{b}_{3}}{\longmapsto}\left(1+4 a t(1+w)^{-5} x^{3} z^{4}\right) \cdot x .
\end{aligned}
$$

Here we used $\left(1+a t(1+w)^{-5} x^{3} z^{4}\right)^{4}=1+4 a t(1+w)^{-5} x^{3} z^{4}$ modulo $(x, y, z)^{10}$. The second term has to cancel with $-700(1+w)^{-1} t x^{3} z^{4}$, which leads to $a=175(1+w)^{4}$.

Said differently, we just have to commute the wall automorphism past all the automorphisms of slabs in counterclockwise direction up to the reference cell. For the wall labelled $10 a$ there is only one such slab, $\mathfrak{b}_{3}$, with normal vector $(-3,2)$, and the monomial is $x^{3} z^{4}=z^{(1,4,0,4)}$. The application of the associated automorphism yields the power $-\langle(-3,2),(1,4)\rangle=-5$ of $1+w$. The coefficient $4=-\langle(4,-1),(-1,0)\rangle$ is picked up from passing $x$ past the wall in question. By the form of the automorphisms, looking at $\overline{\theta_{v}^{k}}(y)$ or $\overline{\theta_{v}^{k}}(z)$ gives the same result. This is a consistency check.

The other coefficients can be computed analogously, giving

$$
1+175(1+w)^{4} t x^{3} z^{4}, \quad 1+14(1+w)^{7} t^{2} x^{3} z, \quad 1-56(1+w)^{4} t^{2} w x^{2} y^{2}
$$

for the functions associated to the walls $10 a, 10 b$ and $10 c$, respectively. 
Continuing in this fashion we can insert new walls essentially uniquely to make the gluing consistent to any finite $\tau$-order.

REMARK 4.2. 1) There is one miracle here. Namely, while the scattering computation works only after localization at $1+w$, no negative powers of $1+w$ ever have to be inserted. This is indispensable because walls and slabs can only carry regular functions. For general slab functions and slab configurations this might indeed not be true, see the discussion in $\S 4.4 .1$ of [GrSi2]. So we need a condition, and the condition we found to make it work ([GrSi2 $]$, Definition 1.25 ,ii) morally says that the deformation problem has a unique solution locally in codimension 2 up to isomorphism. This is a natural condition. Nevertheless, the proof that no denominators occur in $\S 4.4$ of $[\mathbf{G r S i 2}]$ is the most technical part of the story. We refer to $[\mathbf{G r S i 2}]$ for a more complete discussion.

2) For $\operatorname{dim} \sigma_{\mathfrak{j}} \in\{n-1, n\}$ the algorithm is the same, but there are higher degree monomials with vanishing $\sigma_{\mathfrak{j}}$-order. In the $(n-1)$-dimensional case a perturbation argument as in $§ 4.4$ suffices to show that one can achieve consistency without changing the slab functions and without introducing denominators, see [GrSi2] §4.3. In the $n$-dimensional case this is not an issue because there are no slabs and hence there are no denominators and all monomials have positive order anyway. This last case is essentially the situation that was treated by Kontsevich and Soibelman in [KoSo]. In essence, our approach trades the difficult gradient flow arguments in [KoSo] for algebraic arguments for the $\operatorname{dim} \sigma_{\mathfrak{j}}=n-1$ case, while the $\operatorname{dim} \sigma_{\mathfrak{j}}=n-2$ case is only non-trivial for $n \geq 3$ and is the most difficult issue.

4.4. The perturbation idea. Sometimes a simple geometric perturbation of the starting data allows one to simplify a scattering situation. In [GrSi2], $\$ 4.2$ we formalize this in the notion of infinitesimal scattering diagrams. Here we just want to illustrate the concept by one simple example.

EXAMPLE 4.3. Consider the two-dimensional example of a local scattering situation with $x y z=t$ shown in Figure 4.4. By formally perturbing the slabs we arrive at Figure 4.5 on the left. The functions are the slab functions on the various polyhedral pieces. While this is not a legal polyhedral decomposition, we can still insert walls inductively to make the diagram consistent to any finite order. The result is shown
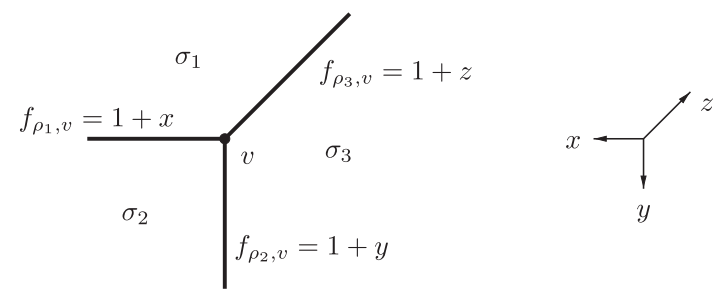

FiguRE 4.4. Scattering of three non-trivial slabs. 


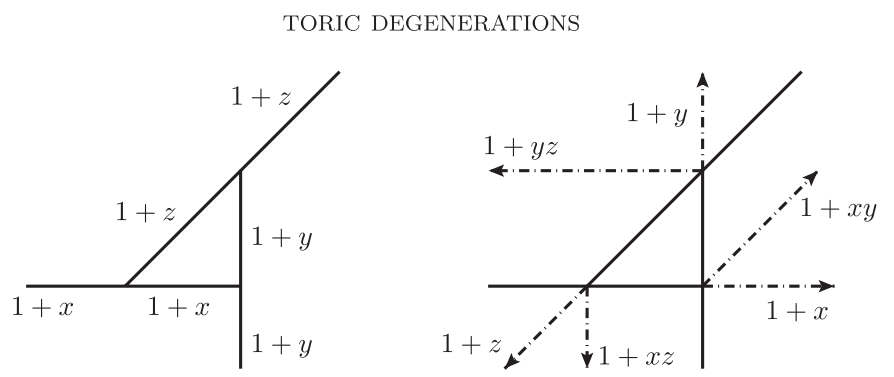

FiguRE 4.5. Perturbed scattering diagram.

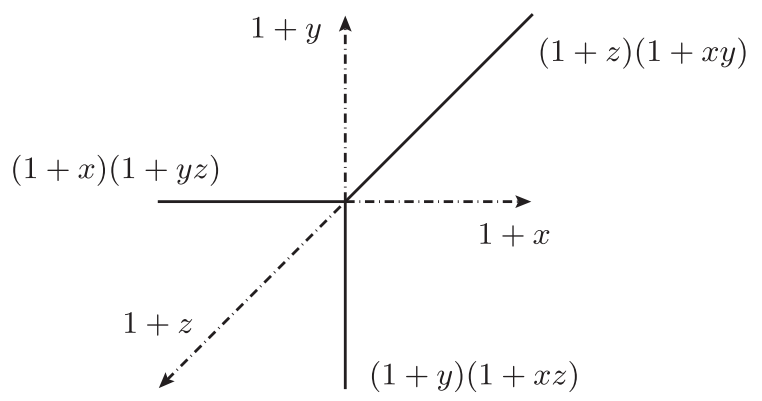

FiguRE 4.6. Result of collapsing Figure 4.5, right.

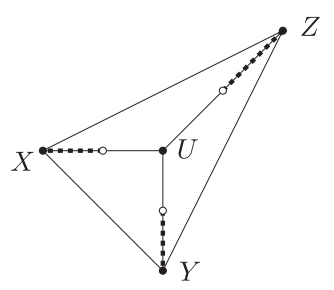

FiguRE 4.7. A tropical manifold for a degeneration of cubic surfaces.

in Figure 4.5 on the right. Note that this diagram is entirely determined by the most simple scattering situation of $\S 4.1$ via affine isomorphisms.

Now the point of this discussion is that we can collapse this diagram again by superimposing walls and slabs with the same direction if necessary. For the present situation the result is shown in Figure 4.6. This configuration of slabs and walls is consistent to all orders.

We can also make this example projective. The smallest polarization produces a degeneration of cubic surfaces as follows.

EXAmPle 4.4. Consider the tropical manifold depicted in Figure 4.7, with three unit triangles as maximal cells and one focus-focus singularity on each of the three interior edges. The piecewise affine function is defined by the values $0,0,0,1$ on the 4 vertices $U, X, Y, Z$, respectively. The vertices also define homogeneous coordinates 
written by the same symbols. This exhibits $X_{0}$ as the union of hyperplanes $V(X Y Z) \subseteq$ $\mathbb{P}^{3}$. Dehomogenizing with respect to $U$ gives the affine coordinates $x_{0}=X / U, y_{0}=$ $Y / U, z_{0}=Z / U$, and in these coordinates we define the slab functions by

$$
f_{\rho_{1}, U}=1+x_{0}, \quad f_{\rho_{2}, U}=1+y_{0}, \quad f_{\rho_{3}, U}=1+z_{0} .
$$

Let us compute an affine chart for the deformation. To this end we look at Figure 4.6 and perform a computation similar to the basic gluing computation in $§ 2.3$. As reference chamber choose the upper left quadrant in Figure 4.6. In this chamber $x=X / U$ is the uncorrected toric coordinate $x_{0}$, but $y=Y / U$ and $z=Z / U$ receive corrections from crossing walls and slabs. For $z$ we need to cross the wall with function $1+y_{0}$, while for $y$ there is the wall with function $1+z_{0}$ and the slab with function $\left(1+x_{0}\right)\left(1+y_{0} z_{0}\right)$. The complete list of relations therefore is

$$
\begin{aligned}
x_{0} y_{0} z_{0} & =t \\
x & =x_{0} \\
z & =\left(1+y_{0}\right) z_{0} \\
y & =\left(1+x_{0}+z_{0}+y_{0} z_{0}+x_{0} y_{0} z_{0}\right) y_{0} .
\end{aligned}
$$

Eliminating $x_{0}, y_{0}, z_{0}$ exhibits an affine chart for the deformation as the hypersurface

$$
x y z=t((1+t)+x+y+z) .
$$

in $\mathbb{A}^{4}$ with coordinates $x, y, z, t$. Homogenizing yields the degeneration of singular cubic surfaces

$$
X Y Z=t\left((1+t) U^{3}+(X+Y+Z) U^{2}\right) .
$$

The general fibre has 3 ordinary double points at $[1,0,0,0],[0,1,0,0]$ and $[0,0,1,0]$; these come from the vertices $X, Y, Z$ on the boundary of $B$.

\section{Three-dimensional examples}

So far we have essentially considered two-dimensional examples, the only exception being the three-dimensional sample scattering computation in $\S 4$.3. We already observed in this example one complication in higher dimensions, the potential presence of poles in the scattering procedure, see Remark 4.2,(1) for a discussion how this is handled. We also observed in this example that the scattering procedure generally requires higher order corrections to the slab functions, and the slab functions themselves propagate. In two dimensions ad hoc solutions can be used to do this. In higher dimensions this propagation is more complicated and we need a homological argument ([GrSi2], §3.5). Another, more fundamental difference in higher dimensions is the fact that the codimension two intersection loci of walls and slabs, the joints, are higher dimensional. Thus in the automorphism $\overline{\theta_{j}}$ associated to a loop around a joint $\mathfrak{j}$ there may be monomials tangent to $\mathfrak{j}$. These can not be removed by inserting walls.

It nevertheless turns out that once we have run the homological argument, this only happens for joints $\mathfrak{j}$ contained in a codimension two cell $\sigma_{\mathfrak{j}}$ of $\mathscr{P}$, and the remaining terms are either undirectional (pure $t$-powers, $t^{l}$ ) or of the form $t^{k} z^{m_{v v^{\prime}}^{\rho}} / f_{\rho, v}$ 

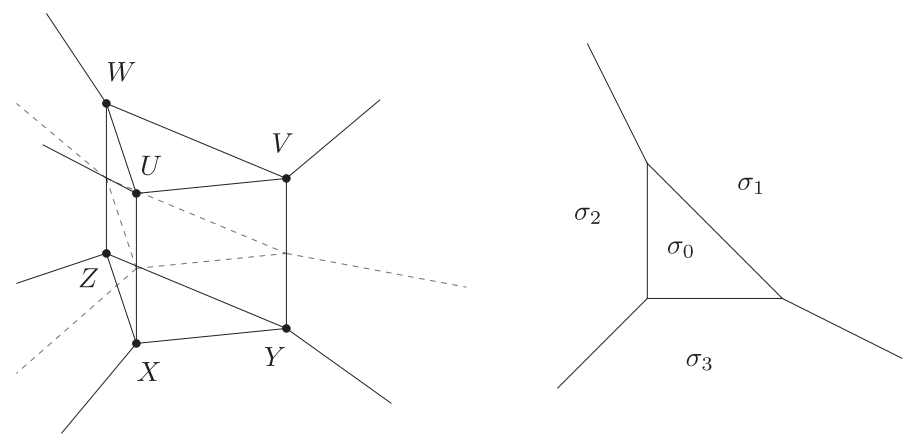

FiguRE 5.1. The tropical manifold for a degeneration of $K_{\mathbb{P}^{2}}$ (left) and its projection to the plane (right).

([GrSi2], Proposition 3.23). Here $m_{v v^{\prime}}^{\rho}$ is defined by affine monodromy as in (2.2). In other words, these terms arise from pure $t$-powers on some other affine chart. The final step in our algorithm uses a normalization procedure to get rid of these terms also ([GrSi2], §3.6). The normalization essentially says that the logarithms of the slab functions do not contain pure $t$-powers. Remarkably, this step not only makes sure that no obstructions arise in the deformation process, but also makes $t$ a canonical parameter in the sense of mirror symmetry, traditionally denoted $q$.

Examples featuring all phenomena in this process are very complicated to run through explicitly. We therefore content ourselves with one non-compact example treating a degeneration of the total space of $K_{\mathbb{P}^{2}}$, the so-called "local $\mathbb{P}^{2}$ " from the mirror symmetry literature [ChKlYa], and its mirror.

Example 5.1. Consider the tropical manifold $B$ shown in Figure 5.1. There is a total of six maximal cells $\sigma_{0}, \ldots, \sigma_{5}$ with only $\sigma_{0}$ bounded, a prism. The maximal cells adjacent to the sides of the prism are $\sigma_{1}, \sigma_{2}, \sigma_{3}$ as shown in the figure. The remaining $\sigma_{4}, \sigma_{5}$ are adjacent to the bottom and top of $\sigma_{0}$, respectively. The affine structure is then completely determined by

$$
\sigma_{0}=\operatorname{conv}\{(0,0,0),(1,0,0),(0,1,0),(0,0,1),(1,0,1),(0,1,1)\},
$$

by the directions $(2,-1, \pm 1),(-1,2, \pm 1),(-1,-1, \pm 1)$ of the unbounded edges in a chart on the upper (plus sign) and lower (minus sign) half, and by requiring the monodromy around the discriminant locus (the dashed lines in the figure) to be primitive and positive. Note that $(0,0,1)$ is an invariant tangent vector defining an affine projection as suggested in Figure 5.1 on the right. As piecewise affine function $\varphi$ we take a minimal one changing slope by 1 along each codimension one cell and vanishing on $\sigma_{0}$. In particular, on the six unbounded edges $\varphi$ takes the value 1 at the first integral point different from a vertex.

The nontrivial slabs $\left(f_{\mathfrak{b}} \neq 1\right)$ are the six vertical cells of codimension one. If we denote by $s$ the monomial with direction $(0,0,-1)$ and with $\sigma_{0}$-order 0 and by $s^{\prime}$ its inverse, the gluing functions are

$$
f_{\mathfrak{b}}=1+s \text { (upper half), } f_{\mathfrak{b}}=1+s^{\prime} \text { (lower half). }
$$



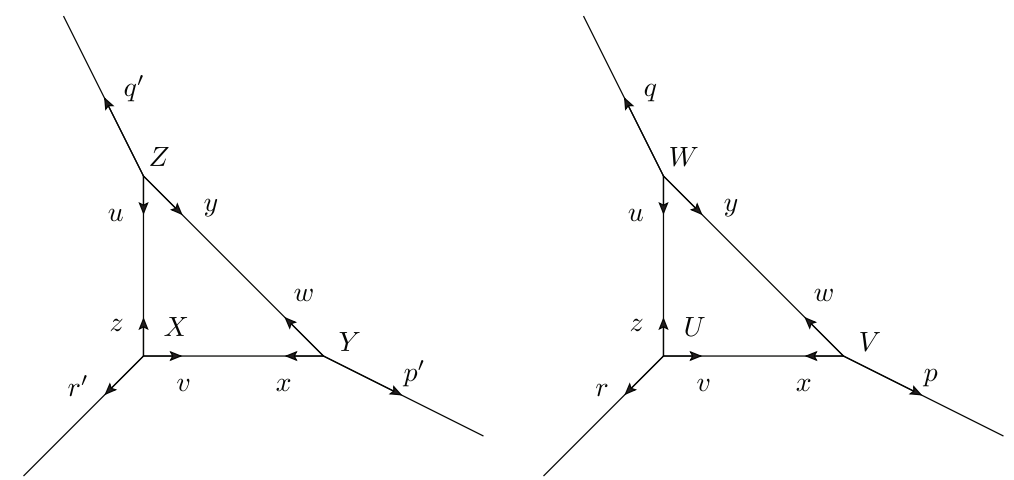

FiguRE 5.2. The generators of the affine patches (left: bottom, right: top).

Note that by (4.2) and the change of vertex formula (2.3) any one slab function determines all the others.

This structure is already consistent along the three vertical edges of the prism. To get consistency everywhere it only remains to insert six vertical walls in $\sigma_{4}$ and $\sigma_{5}$ each. These extend the six vertical codimension one cells to infinity. There is no scattering because the monomials carried by the walls and slabs all point in the same direction $(0,0,1)$, the invariant tangent vector, and hence the corresponding automorphisms mutually commute.

To study this example it is not advisable to write down the homogeneous coordinate ring. In fact, because this is a non-compact example it does not suffice to take the six vertices $X, Y, Z, U, V, W$ as generators of a homogeneous coordinate ring. Rather we need a number of generators of degree 0 defined by tangent vectors in the unbounded directions. Due to the non-simplicial nature of the polyhedra this leads to a long list of generators and an even longer list of relations.

Instead we use the construction via gluing affine patches from $[\mathbf{G r S i 2}]$. There are six vertices, and correspondingly we have a cover of the degeneration by six affine open sets. Figure 5.2 shows our choices of generators, where the arrows should be thought of as tangent to edges. Thus in a chart at $U$ the generating monomials are

$$
v=z^{(1,0,0,0)}, z=z^{(0,1,0,0)}, r=z^{(-1,-1,1,1)}, s=z^{(0,0,-1,0)}, t=z^{(0,0,0,1)},
$$

with single relation

$$
r v z s=t,
$$

a semi-stable (normal crossings) degeneration. Because of the symmetry of the example the situation is analogous in the five other charts.

Now we have to adjust these local models by the slab functions. A computation analogous to $\S 2.3$ gives

$$
\begin{aligned}
& \text { rvzs }=(1+s) t(\text { at } U), \quad \text { pwxs }=(1+s) t(\text { at } V), \quad \text { quys }=(1+s) t(\text { at } W), \\
& r^{\prime} v z s^{\prime}=\left(1+s^{\prime}\right) t(\text { at } X), \quad p^{\prime} w x s^{\prime}=\left(1+s^{\prime}\right) t(\text { at } Y), \quad q^{\prime} u y s^{\prime}=\left(1+s^{\prime}\right) t(\text { at } Z) \text {. }
\end{aligned}
$$

Here the variables take reference to any maximal cell containing them. Thus $x, y$, $z, u, v, w$ can all be thought of as monomials on $\sigma_{0}$, while for example $r$ is a monomial 
on $\sigma_{2}$ or $\sigma_{3}$. The patching between these charts is given by affine geometry in the relevant maximal cells. In particular, variables with the same name are all identified, and we have relations such as

$$
x=v^{-1}, \quad u=x w^{-1}, \quad s^{\prime}=s^{-1}, \quad q=p w^{3} \quad \text { etc. }
$$

As a consistency check one can verify compatibility of the relations with the gluing. For example, going from the chart at $U$ to the chart at $V$ means the substitution

$$
v \mapsto x^{-1}, \quad z \mapsto x^{-1} w, \quad r \mapsto x^{3} p, \quad s \mapsto s .
$$

This maps the relation rvzs $=(1+s) t$ to pwxs $=(1+s) t$, as expected. Similarly, to go from $U$ to $X$ means substituting

$$
v \mapsto v, \quad z \mapsto z, \quad r \mapsto r^{\prime} s^{\prime}, \quad s \mapsto\left(s^{\prime}\right)^{-1}
$$

into $r v z s=(1+s) t$, leading to $r^{\prime} v z s^{\prime}=\left(1+s^{\prime}\right) t$.

At this point we have written down a degeneration $\pi: X \rightarrow \mathbb{A}^{1}=\operatorname{Spec} \mathbb{C}[t]$ with $X$ covered by six affine open sets. We claim that a general fibre $X_{t}$ is an open subset of the total space $K_{\mathbb{P}^{2}}$ of the canonical bundle of $\mathbb{P}^{2}$. To this end fix $t \in \mathbb{C} \backslash\{0\}$ and define a projection

$$
\kappa: X_{t} \longrightarrow \mathbb{P}^{2}
$$

by viewing the triples $X, Y, Z$ or $U, V, W$ as homogeneous coordinates on $\mathbb{P}^{2}$. Thus set-theoretically the restriction of $\kappa$ to the chart at $U$ is

$$
(r, v, z, s) \longmapsto[1, v, z] .
$$

It is straightforward to check compatibility with the patching. For example, in the intersection with the chart at $V$ we find

$$
\kappa(p, w, x, s)=[x, 1, w]=\left[v^{-1}, 1, v^{-1} z\right]=[1, v, z] .
$$

Analogous computations show compatibility on the ring level.

The fibre of $\kappa$ over a closed point of $\mathbb{P}^{2}$, say $[1, v, z]$, is the hypersurface rvzs $(1+s) t=0$ in $\mathbb{A}^{2}=\operatorname{Spec}(\mathbb{C}[r, s])$. Note that $X_{t}$ is disjoint from $s=0$ or from $s^{\prime}=0$ since $t \neq 0$, so it suffices to work in one chart only. If $v z \neq 0$ this is a hyperbola, hence isomorphic to $\mathbb{A}^{1} \backslash\{0\}$. On the other hand, if $v z=0$ we must have $s=-1$ and $r$ has no restrictions, so this is an $\mathbb{A}^{1}$. The global meaning of this comes by observing that the fibre coordinates $p, q, r$ transform dually to the sections $d x \wedge d w, d y \wedge d u$ and $d z \wedge d v$ of $K_{\mathbb{P}}^{2}$. For example, since $y=z^{-1} v$ and $u=z^{-1}$,

$$
q d y \wedge d u=q\left(-z^{-2} v d z+z^{-1} d v\right) \wedge\left(-z^{-2} d z\right)=q z^{-3} d z \wedge d v=r d z \wedge d v .
$$

Above we computed the fibre of $\kappa$ over $[1, v, z]$ to be given by $(r v z-t) s=t$. For given $r, v, z$ this has a solution $s$ as long as $r v z-t \neq 0$. Thus $r v z-t=0$ describes the hypersurface locally that is being removed from $K_{\mathbb{P}^{2}}$ to obtain $X_{t}$. Globally we are removing the graph of a rational section of $K_{\mathbb{P}^{2}}$ with poles along the toric divisor $V(X Y Z)$. 

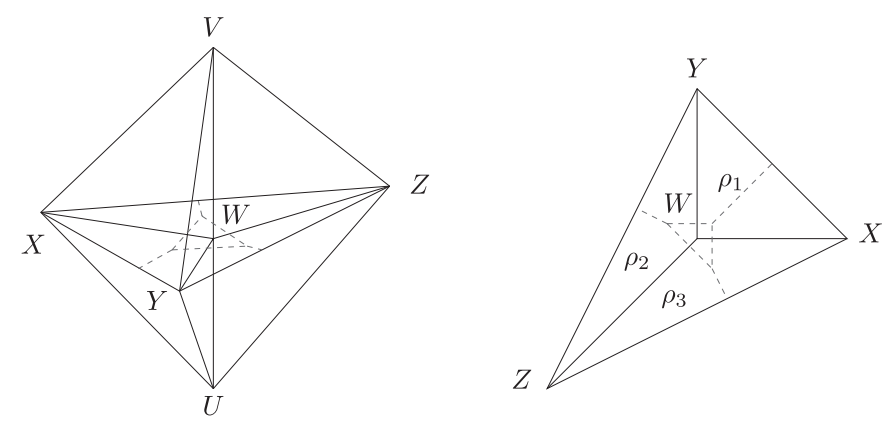

Figure 5.3. The tropical manifold for the mirror of Example 5.1 (left) and the central triangle containing the discriminant locus (right).

EXAMPLE 5.2. An example with somewhat complementary features to Example 5.1 is provided by the mirror. Following the general recipe of [GrSi1] the tropical manifold together with the (multi-valued) function $\varphi$ is obtained by a discrete version of the Legendre transform ([GrSi1], Construction 1.15). By this construction the polyhedral decompositions of a tropical manifold and its mirror are combinatorially dual to each other. As this is not primarily a paper about mirror symmetry we do not explain this construction, but only state the result.

As shown in Figure 5.3 the tropical manifold is a double tetrahedron glued from six standard simplices. We have five exterior vertices labelled $X, Y, Z, U, V$ and one interior vertex $W$. In an affine chart at $W$ the five emanating edges point in the directions $(0,0, \pm 1),(1,0,0),(0,1,0),(-1,-1,0)$. The affine structure is then completely determined by requiring the monodromy around the edges of the discriminant locus (dashed in the figure) to be primitive and positive. The function $\varphi$ is single-valued and can be taken to take value 0 on $X, Y, U, W$ and value 1 on $Z$ and $V$. This again has the property to change slope by 1 along each cell of codimension one.

There are three non-trivial slabs, the three horizontal triangles containing the discriminant locus. Up to automorphisms there is only one set of gluing functions possible at order 0 , namely, in affine coordinates $x=X / W, y=Y / W, z=Z / W$ at $W$ :

$$
f_{\rho_{1}, W}=1+x+y, \quad f_{\rho_{2}, W}=1+y+z, \quad f_{\rho_{3}, W}=1+z+x .
$$

The expressions at the other vertices follow from this by the change of vertex formula (2.3).

To make this structure consistent to all orders only requires propagating the slab functions to the neighbouring slabs. This leads to

$$
f_{\mathfrak{b}, W}=1+x+y+z,
$$

for any of the three slabs $\mathfrak{b}=\rho_{i}$.

The approach by homogeneous coordinates works well again in this case. We get the toric relation

$$
X Y Z=t W^{3}
$$


and the homogenization of the gluing relation $u v=(1+x+y+z) t$ gives

$$
U V=t^{2}(X+Y+Z+W) W .
$$

As one checks in local coordinates this is a complete set of relations. For $t \neq 0$ the projective variety $X_{t}$ is a conic bundle over $\operatorname{Proj}\left(\mathbb{C}[X, Y, Z, W] /\left(X Y Z-t W^{3}\right)\right)$ with singular fibers over $(X+Y+Z+W) W=0$. This base space of the conic bundle is the quotient of $\mathbb{P}^{2}$ by the $\mathbb{Z} / 3$-action

$$
\xi \cdot\left[x_{0}, x_{1}, x_{2}\right]=\left[\xi x_{0}, \xi^{2} x_{1}, x_{2}\right],
$$

for $\xi$ a primitive third root of unity.

The suggestion in the literature for the mirror to $K_{\mathbb{P}^{2}}$ is to take the family of non-complete Calabi-Yau varieties defined by

$$
u v=1+x+y+t x^{-1} y^{-1}
$$

in $\mathbb{C}^{2} \times\left(\mathbb{C}^{*}\right)^{2}\left[\right.$ ChKlYa]. Here $u, v$ are the coordinates on $\mathbb{C}^{2}$. This is exactly the open subset of our family fibering over the big cell of the weighted projective space. See [Gr1], $§ 4$ for a discussion how this fits with the SYZ picture of local mirror symmetry.

As written this family does not come correctly parametrized for the purpose of mirror symmetry. Rather, a period integral defines a new parameter $q$ related to $t$ by the so-called mirror map. It is one striking feature of our approach that this mirror map comes up naturally via the normalization condition. The present example is too local to illustrate the need for doing this, but as mentioned at the beginning of this section, generally our algorithm requires the logarithm of the slab functions to not contain any pure $t$-powers, see $[\mathbf{G r S i 2}], \S 3.6$. In the present example this means adding a power series $g=\sum_{l \geq 0} a_{l} t^{l}$ to $f_{\mathfrak{b}, W}$ with the property that

$$
\log \left(f_{\mathfrak{b}, W}+g\right)=\sum_{k \geq 1} \frac{(-1)^{k+1}}{k}(x+y+z+g(x y z))^{k} \in \mathbb{C} \llbracket x, y, z \rrbracket
$$

does not contain any monomials $(x y z)^{l}=t^{l}$. This condition determines the coefficients $a_{k}$ inductively:

$$
g(t)=-2 t+5 t^{2}-32 t^{3}+286 t^{4}-3038 t^{5}+\cdots
$$

It follows from the period computations in $[\mathbf{G b Z a}]$ that the modified family

$$
X Y Z=t W^{3}, \quad U V=t^{2}(X+Y+Z+(1+g(t)) W) W,
$$

is then indeed written in canonical coordinates, that is, the mirror map becomes trivial.

\section{References}

[ChKlYa] T.M. Chiang, A. Klemm, S.-T. Yau, E. Zaslow: Local mirror symmetry: calculations and interpretations, Adv. Theor. Math. Phys. 3 (1999), 495-565.

[GbZa] T. Graber, E. Zaslow: Open-string Gromov-Witten invariants: calculations and a mirror "theorem". in "Orbifolds in mathematics and physics (Madison, WI, 2001)", 107-121, Contemp. Math., 310, Amer. Math. Soc. 2002. 
[Gr1] M. Gross: Examples of special Lagrangian fibrations, in: Symplectic geometry and mirror symmetry (Seoul, 2000), 81-109, World Sci. Publ. 2001.

[Gr2] M. Gross: Toric Degenerations and Batyrev-Borisov Duality, Math. Ann. 333 (2005), 645-688.

[GrSi1] M. Gross, B. Siebert: Mirror symmetry via logarithmic degeneration data I, J. Differential Geom. 72 (2006), 169-338.

[GrSi2] M. Gross, B. Siebert: From real affine to complex geometry, to appear in Annals of Math.

[Ha] J. Harris: Algebraic geometry, Springer 1992.

[Ho] M. Hochster: Cohen-Macaulay rings, combinatorics and simplicial complexes, in: Ring theory II, B.R. McDonald, R.A. Morris (eds.), Lecture Notes in Pure and Appl. Math. 26, M. Dekker 1977.

[KoSo] M. Kontsevich, Y. Soibelman: Affine structures and non-Archimedean analytic spaces, in: The unity of mathematics (P. Etingof, V. Retakh, I.M. Singer, eds.), 321-385, Progr. Math. 244, Birkhäuser 2006.

[St] R. Stanley: Combinatorics and commutative algebra, Second ed., Birkhäuser 1996.

[Sy] M. Symington: Four dimensions from two in symplectic topology, in: Topology and geometry of manifolds (Athens, GA, 2001), 153-208, Proc. Sympos. Pure Math. 71, Amer. Math. Soc. 2003.

[Wi] J. Williamson: On the algebraic problem concerning the normal forms of linear dynamical systems, Amer. J. Math. 58 (1936), 141-163.

UCSD Mathematics, 9500 Gilman Drive, La Jolla, CA 92093-0112, USA

E-mail address: mgross@math.ucsd.edu

Department Mathematik, Universität Hamburg, Bundesstrasse 55, 20146 Hamburg, GERMANY

E-mail address: bernd.siebert@math.uni-hamburg.de 